\title{
Chaos from Switched-Capacitor Circuits: Discrete Maps
}

\author{
ANGEL RODRIGUEZ-VAZQUEZ, MEMBER, IEEE, JOSE L. HUERTAS, MEMBER, IEEE, \\ ADORACION RUEDA, BELEN PEREZ-VERDU, AND LEON O. CHUA, FELLOW IEEE
}

Invited Paper

\begin{abstract}
A special-purpose analog computer made of switched-capacitor circuits is presented for analyzing chaos and bifurcation phenomena in nonlinear discrete dynamical systems modeled by discrete maps

$$
x_{n+1}=f\left(x_{n}\right) \text {. }
$$
\end{abstract}

Experimental results are given for four switched-capacitor circuits described by well-known discrete maps; namely, the logistic map, the piecewise-finear unimodal (one-hump) map, the Hénon map, and the Lozi map.

\section{INTRODUCTION}

Chaos has been widely observed in numerous discrete dynamical systems modeled by a discrete map [1]-[4]

$$
x_{n+1}=f\left(x_{n}\right) \text {. }
$$

Such discrete maps occur naturally in many physical (biology, chemistry, engineering, physics, etc.), social, and economical systems. They also arise frequently in the analysis of systems modeled by ordinary differential equations via their Poincaré maps-an indispensable tool in any rigorous study of chaos.

Until recently, discrete maps were simulated using digital computers. Since the objective of such simulation is usually to investigate the system's asymptotic behavior after the initial transients have decayed to zero-henceforth referred to as the steady state in this paper-a large number of numerical iterations must be performed for each given initial condition and system parameters. This is an extremely time-consuming task which severely restricts a detailed bifurcation analysis to at most two parameters; and any for low-order (less than two) discrete maps.

Manuscript received September 3, 1986; revised December 16, 1986. This research was partially supported by the Office of Naval Research under Contract N00014-86-K-0351 and by the National Science Foundation under Grant MIP-8614000.

A. Rodriguez-Vazquez, J. L. Huertas, A. Rueda, and B. Perez-Verdu are with the Departamento de Electricidad y Electronica, Facultad de Fisica, Universidad de Sevilla, Seville, Spain.

L. O. Chua is with the Department of Electrical Engineering and Computer Sciences, University of California, Berkeley, CA 94720, USA.

IEEE Log Number 8714774.
Our objective in this paper is to present a much more efficient approach for simulating discrete maps using switched-capacitor circuits. Since the simulation of such circuits gives real-time solutions, it is ideally suited for carrying out a comprehensive multi-parameter bifurcation analysis. Such switched-capacitor circuits represent therefore a special-purpose analog computer for simulating chaotic and bifurcation phenomena.

\section{Definitions, Properties, and Examples of Discrete MAPS}

Consider a real closed interval $I=[a, b]$ and a nonlinear function $f(\cdot)$ which transforms any point $x$ of $l$ into some point $x^{\prime}$ in the same interval

$$
f: l \rightarrow l \text {. }
$$

Choose an arbitrary initial point $x_{0}$ in $/$ and iterate it via the algorithm

$$
x_{n+1}=f\left(x_{n}\right), \quad n=0,1,2, \cdots .
$$

Equation (1) is called a discrete map of the interval $I$. We can generalize this "scalar" discrete map (1) into an $m$ dimensional interval map via the vector equation

$$
x_{n+1}=f\left(x_{n}\right)
$$

where $x_{n+1}$ and $x_{n}$ are $m$-dimensional vectors and $f(\cdot)$ is a nonlinear vector function of $x_{n}$. Each component $x_{n ;}$ of $x_{n}$ assumes values from some closed interval $l_{i}=\left[a_{i}, b_{i}\right]$.

We can interpret (1) and (2) as a dynamical system where $n$ plays the role of the time variable. In spite of their structural simplicity, the dynamics of many simple nonlinear discrete maps is extremely rich and complicated. They can possess stable fixed points ${ }^{1}$, periodic orbits of different periodicity, and "chaotic" regimes. Moreover, all of these behaviors can be observed in a parameter-dependent family of maps over various nonempty parameter ranges.

\footnotetext{
${ }^{1} A$ point $x^{*}$ is called a fixed point of the map $f$ iff $x^{*}=f\left(x^{*}\right)$.
} 


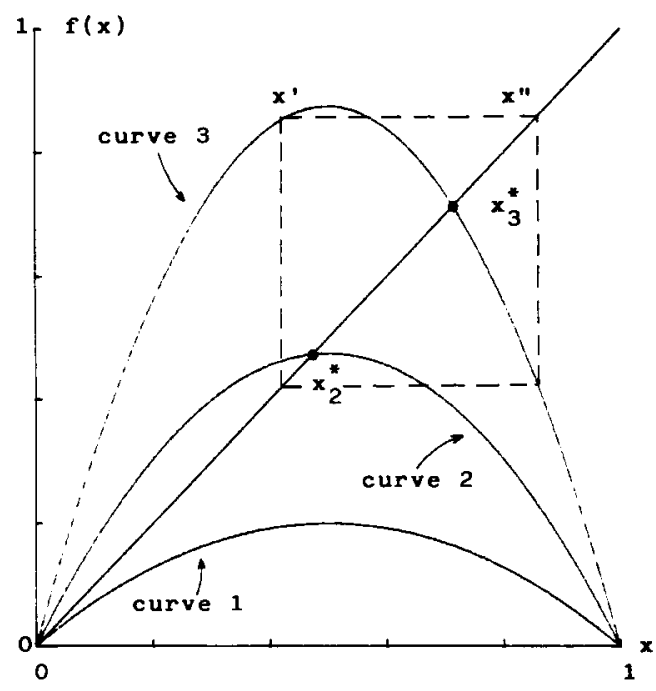

(a)

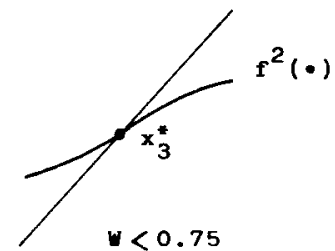

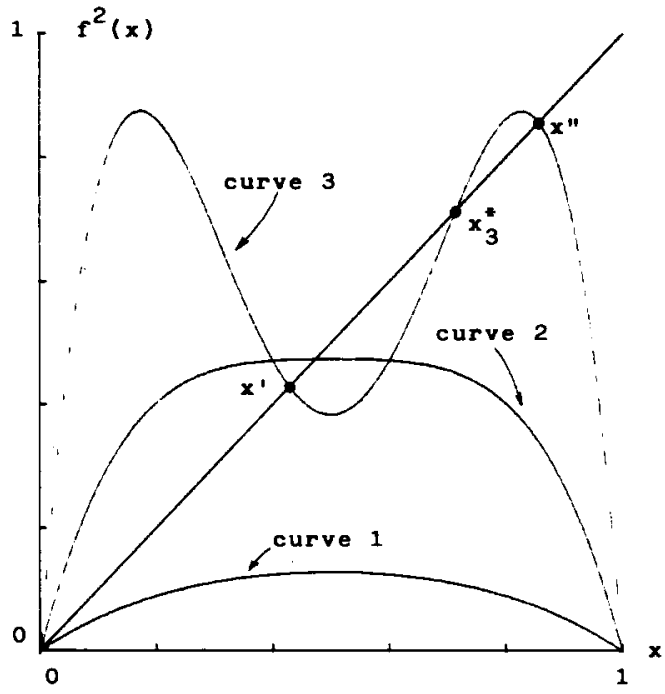

(b)

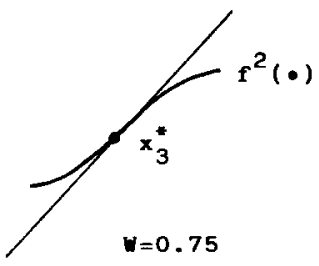

(c)

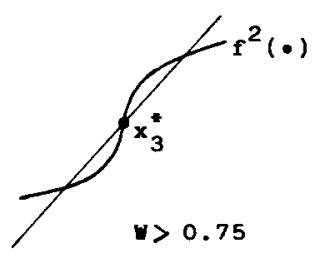

Fig. 1. (a) Fixed points of $f(x)$ for $W<0.25$ (curve 1), $0.25<W<0.75$ (curve 2), and $W>0.75$ (curve 3). (b) Corresponding fixed points for $f^{2}(x)=f[f(x)]$. (c) Bifurcation of $f^{2}(x)$ gives rise to two new stable fixed points.

For simplicity, consider first the following standard example of a one-dimensional map; namely, the logistic map:

$$
x_{n+1}=4 W x_{n}\left(1-x_{n}\right)=f\left(x_{n} ; W\right)
$$

where $I=[0,1]$ and $W$ is a parameter which varies from 0 to 1. The logistic map is an example of a one-parameter family of discrete maps which transforms (or maps) the interval $l$ into itself.

The map $f(x ; W)$ is shown in Fig. $1(a)$ for three different values of $W$. Note that for $0<W<0.25$, this map has only one fixed point, located at $x=0$. For $0.25<W<1, f(\cdot)$ has two fixed points, at $x=0$ and $x=(1-0.25 / W)=x^{*}$. The fixed point $x=0$ is stable for $0<W<0.25$, and unstable for $0.25<W<1$. On the other hand, the fixed point $x^{*}$ is stable for $0.25<W<0.75$, and unstable for $0.75<W<$ 1. Hence the map $f(x ; W)$ has one stable fixed point for $0<W<0.75$, and no stable fixed points for $0.75<W<$ 1.

To illustrate the "stability" of the above fixed points for a particular value of $W$, choose any $x_{0} \neq 0$ and iterate the map repeatedly. After waiting for a time large enough to ensure that any transients have decayed nearly to zero, we plot a point for every subsequent iterated value of $x_{n}$. By repeating this iteration for different values of $W$ from 0 to 1, we obtain Fig. 2(a). Note that there are several subintervals along the $W$ axis, each one corresponding to a different qualitative behavior. The interval between 0 and 0.25 in Fig. 2(a) corresponds to those maps with only one stable fixed point. Curve $1(W<0.25)$ in Fig. $1(a)$, is an example of such maps. For any initial condition, the solution tends to the fixed point at $x=0$ and remains there. For curve 2 in Fig. $1(a)$, and for any initial value not equal to zero, $x_{n}$ tends to $x_{2}^{*}$ as $n$ increases. Thus $x_{2}^{*}$ is stable. Maps like the one associated with curve 2 give rise to the parabolic segment between 0.25 and 0.75 in Fig. 2(a). Increasing $W$ beyond 0.75 , we observe that both fixed points become unstable and the associated transient behavior near $x_{3}^{*}$ depends on the value of $W$. Let us begin by assuming a value slightly larger than 0.75 in Fig. 2(a). Here, we observe that for any initial point other than zero, $x_{n}$ does not tend to $x_{3}^{*}$, but rather oscillates back and forth following the rectangular pattern defined by $x^{\prime}$ and $x^{\prime \prime}$, as shown in Fig. 1(a). If point $x^{\prime}$ is reached ${ }^{2}$ at $n=i$, we observe that this point is again reached at instants $i+p$ where $p$ is any even integer, and that point $x^{\prime \prime}$ will be reached at those instants where $p$ is an odd integer. Such a periodic pattern is called a stable period-2 orbit. Maps exhibiting a period-2 orbit give rise to the double-valued region in Fig. 2(a). The transition from the single-valued to the double-valued region in Fig. 2(a) defines a bifurcation point. A geometrical interpretation of this bifurcation is given in Fig. 1(b), where the second iterate of the map, $f^{2}(x) \triangleq f[f(x)]$, is drawn for the same values of $W$ as in Fig. 1 (a). Note that the fixed points of both $f(x)$ and $f^{2}(x)$ are identical for $W<0.75$. As $W$ increases beyond 0.75 , the point $x_{3}^{*}$ becomes unstable in both $f(x)$ and $f^{2}(x)$ and two new sta-

${ }^{2}$ To be precise, $x$ is reached only after an infinite number of iterations. In practice, however, the convergence is extremely fast so that it takes only a finite number of iterations using a computer with a finite word length. 


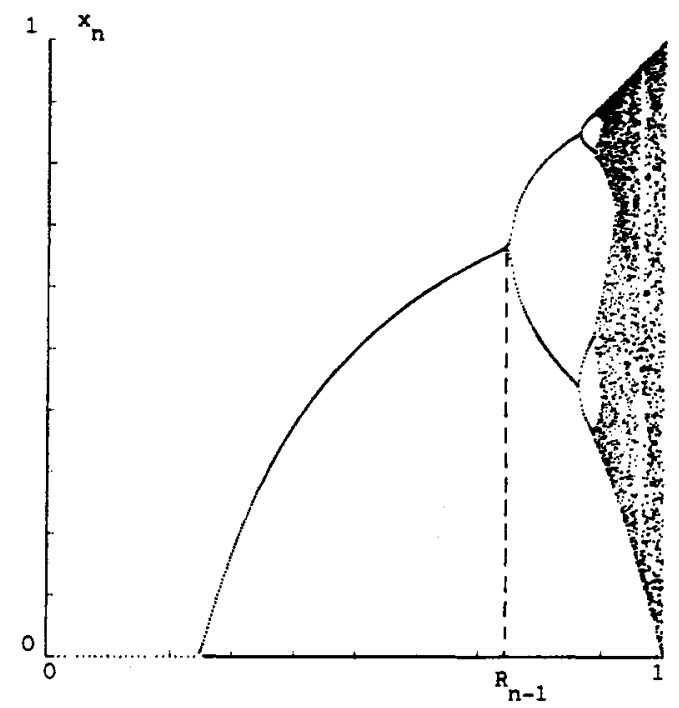

(a)

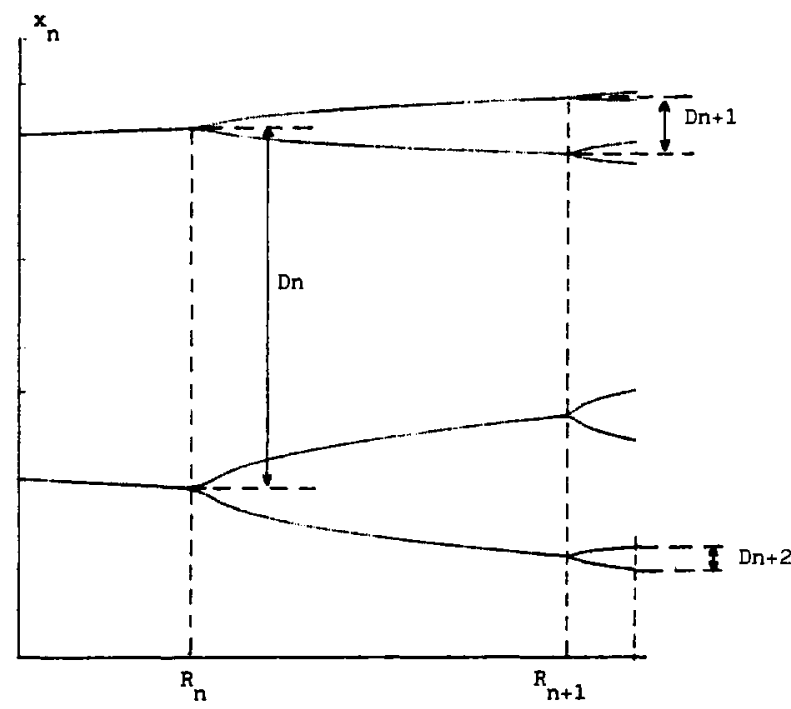

(b)

Fig. 2. (a) "Steady-state" iterates of the logistic map as a function of the parameter $W$. (b) Detailed bifurcation diagram in the vicinity of the period-2 orbit.

ble fixed points $x^{\prime}$ and $x^{\prime \prime}$ bifurcate from $x_{3}^{*}$ and appear in $f^{2}(x)$, as shown in Fig. 1(c). Since these new fixed points of $f^{2}(x)$ are stable, any iteration must converge to one of these fixed points. This corresponds to a period-1 orbit of $f^{2}(\cdot)$, or a period-2 orbit of $f(\cdot)$, since one iteration of $f^{2}(\cdot)$ is equivalent to two iterations of $f(\cdot)$.

If we increase $W$ further, we find that $x_{n}$ converges to orbits of periods equal to a successive power of 2, as shown in Fig. 2(a). This phenomenon is called Period Doubling. When we increase $W$ further from 0.75 , we see that the plot bifurcates successively into two, four, eight, $\cdots$, $2^{n}, \cdots$ branches. Each period- $n$ orbit of $f(\cdot)$ is a period-1 orbit of $f^{n}(\cdot)$, and will henceforth be called a cycle. Each subinterval in the plot in Fig. 2(a) corresponding to a particular period-n orbit, or cycle, is called a "window." Although the period-doubling phenomenon produces an infinite sequence of "cycles," the "width" of the windows progressively diminishes. In fact, a critical point is reached beyond which there are an infinite number of distinct cycles, as well as an uncountable number of initial points that give bounded but totally aperiodic trajectories, generally referred to as chaos.

We can define two important quantities associated with Fig. 2(a). Consider an enlargement in the vicinity of the $n$th window, as shown in Fig. 2(b) and define

$$
A_{n}=\frac{D_{n}}{D_{n+1}}
$$

and

$$
B_{n}=\frac{R_{n}-R_{n-1}}{R_{n+1}-R_{n}}
$$

where $R_{n}, R_{n-1}, R_{n+1}, D_{n}$ and $D_{n+1}$ are defined in Fig. 2(b).

It has been shown in [9] that both ratios approach two universal constants given, respectively, by

$$
A=2.502907870957 \cdots \quad B=4.6692016091029 \cdots .
$$

Within the chaotic region in Fig. 2(a) there exist many small windows corresponding to other periodic orbits, the distinctive feature being that they may correspond to either odd- or even-period orbits. In fact, increasing the parameter beyond a given point, we can find orbits with every integer period. In general, observing the chaotic region is a difficult experimental task, specially if we are interested in the very narrow windows associated with orbits whose period is not a power of two.

Although we have been dealing with the logistic map, the above observations hold for a broad class of one-dimensional maps. Specifically, for those maps where the function $f(\cdot)$ in (1) is a differentiable one-hump (unimodal) function, the same qualitative behavior as the logistic map is observed; namely, the qualitative behavior is essentially independent of the actual form of $f(\cdot)$, it only depends on the value of the parameter. This property is sometimes called "structural universality."

Furthermore, the two quantities $A$ and $B$ obtained above for the logistic map have been found to be "scaling factors" that characterize the onset of chaos through period-doubling for any differentiable unimodal function. This property represents another universal property, sometimes called the "metrical universality."

In contrast to one-dimensional maps, higher dimensional discrete maps can be either conservative (volumepreserving) or dissipative (volume-contracting), invertible or noninvertible. While some results on one-dimensional mappings can be generalized to mappings in $R^{N}$ [3], the latter are usually much richer in their dynamic behavior than the former. For instance, consider the well-known twodimensional Hénon map [25], described by

$$
\begin{aligned}
& x_{n+1}=1-A x_{n}^{2}+y_{n} \\
& y_{n+1}=B x_{n}
\end{aligned}
$$

This map (which reduces to a one-dimensional quadratic map for $B=0$ ) reveals many new phenomena quite typical for multidimensional discrete maps. First, the asymptotic behavior of the system depends on the initial point; i.e., different initial points could give rise to different periodic or aperiodic orbits. This behavior cannot occur in one- 
dimensional unimodal maps, where at most one stable period-1 orbit can exist. Second, for certain parameters and initial points, the system converges to an attractor with a self-similar internal structure [2]. Third, it has been proved that there exist intersections between the stable and unstable manifolds of the Hénon map. These intersections, called homoclinic points, give rise to extremely complicated dynamics, including chaos [4].

We close this section with a selected collection of discrete maps reported in the literature which exhibit chaotic regimes. In particular, Table 1 contains one-dimensional discrete maps reported from many different fields (biology, chemistry, economics, engineering, mathematics, physics, etc.). Note that we can partition Table 1 into three sections. The first five maps correspond to those where "linear operators" and "powers" are required. The next seven maps correspond to those requiring the modulus operator and other piecewise-linear functions. Finally, the last map corresponds to more complex nonlinear operators. Similar groupings also apply for Table 2, where some two-dimensional and three-dimensional maps from different disciplines are collected.

Table 1 A Short Catalog of One-Dimensional Discrete Maps

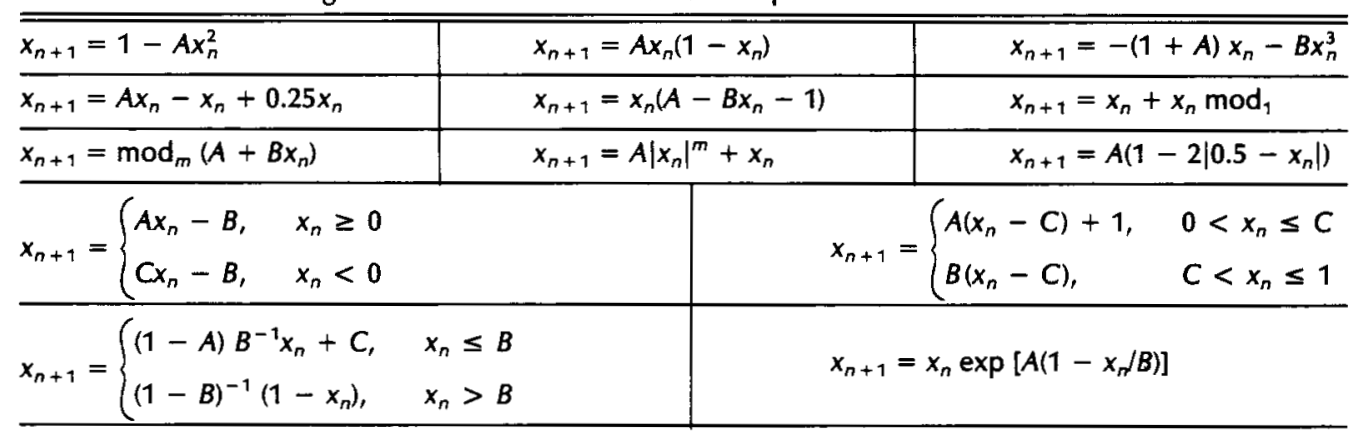

Table 2 A Short Catalog of Two-Dimensional and Three-Dimensional Discrete Maps

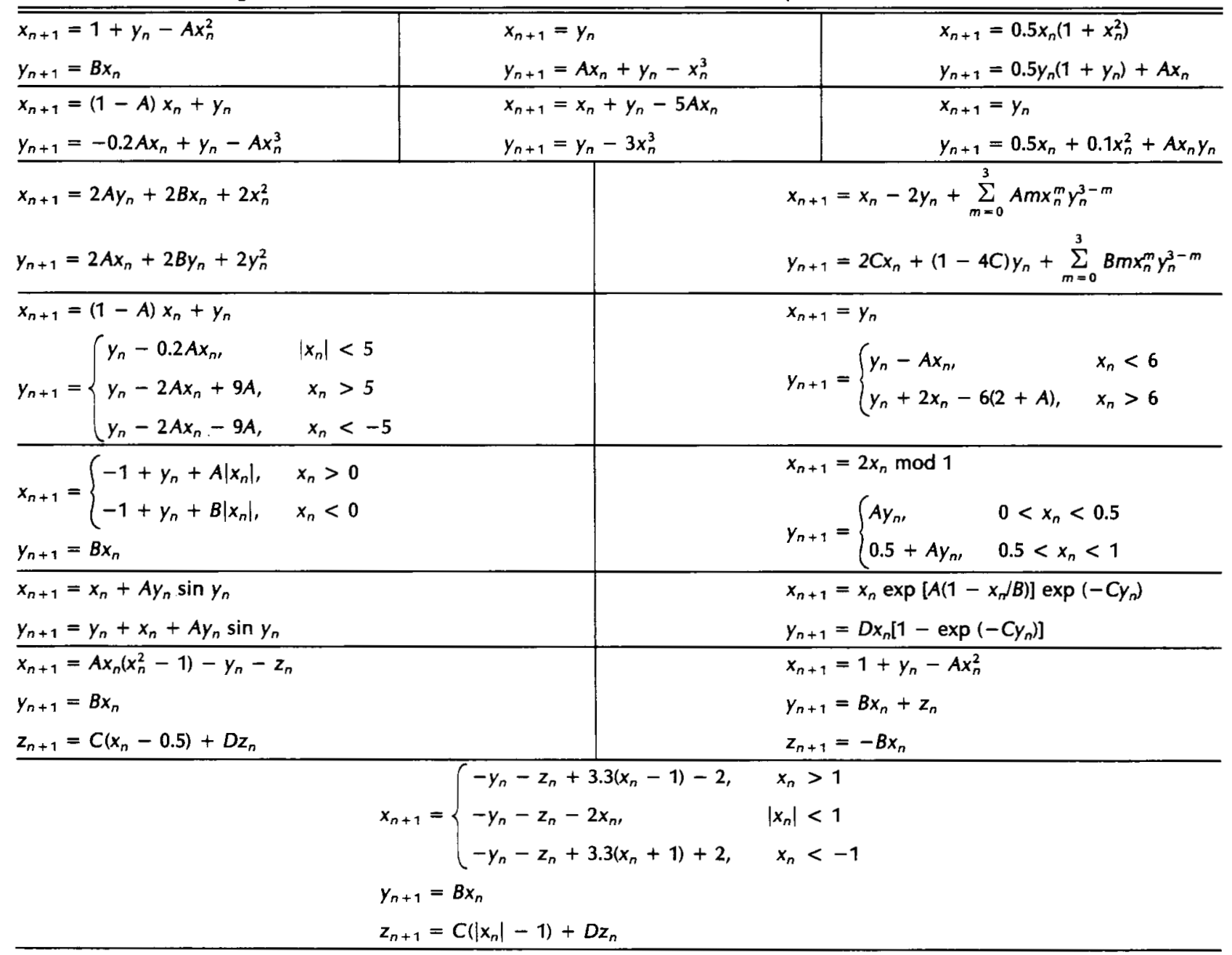


III. Switched-Capacitor (SC) Circuits for Simulating Discrete Maps

\section{A. Circuit Structure}

Fig. 3 shows a simple yet completely general conceptual circuit structure which can be used to synthesize any onedimensional discrete map. Note that the linear blocks con-

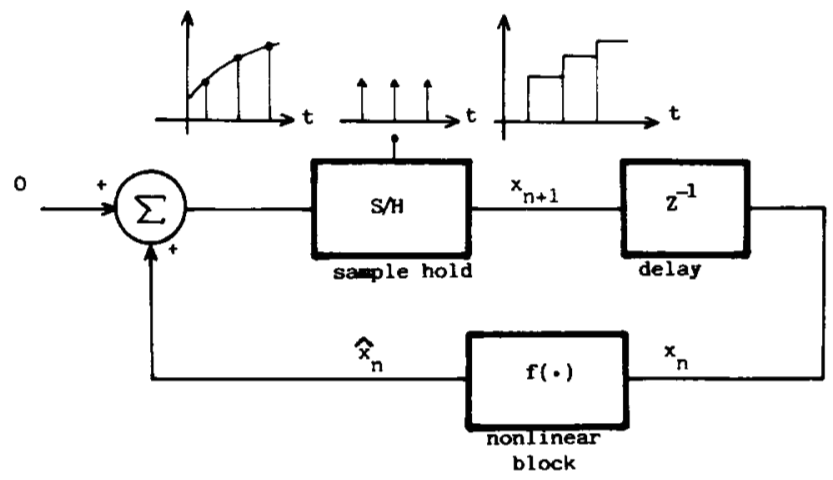

Fig. 3. Conceptual feed back loop for synthesizing any onedimensional discrete map.

sist of the sample-and-hold $(\mathrm{S} / \mathrm{H})$ system and the delay system remains unchanged. Only the nonlinear feedback block for $f(\cdot)$ representing the nonlinear function in (1) needs to be tailored to the discrete map, such as those listed in Table 1. Also, note that the value of the signal $x$ at the $n$ th-time interval is transformed into the signal $\hat{x}$. The output signal of the nonlinear block is first sampled and held, and then applied to the input of a delay block whose output is fed back to the input of the nonlinear block. The clock signal fixing the sampling period determines the iteration pace for the nonlinear feedback loop. The dynamics of this conceptual system is therefore described exactly by (1).

Using switched-capacitor techniques, Fig. 4(a) gives an elegant implementation of the different blocks in Fig. 3. We

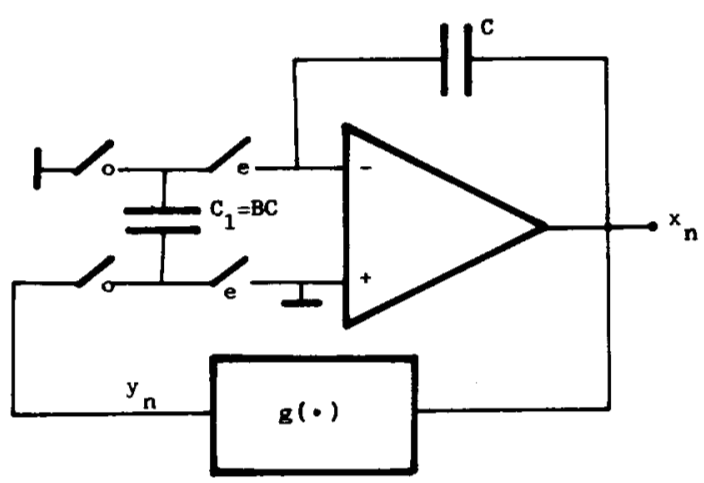

(a)

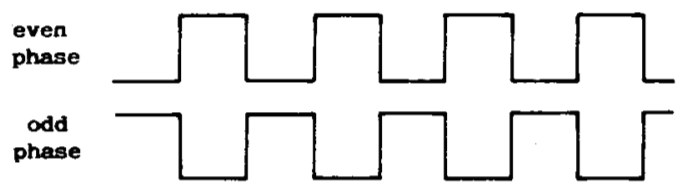

(b)

Fig. 4. (a) SC-integrator nonlinear loop for synthesizing a discrete map. (b) Timing diagram for the switches. can identify a nonlinear block $\mathbf{g}(\cdot)$, an operational amplifier (op-amp), two capacitors, and four analog switches. We assume the switches are controlled by a clock with two nonoverlapping phases whose timing diagram is shown in Fig. 4 (b). Here, the switch labeled " $e$ " (respectively, " $o$ ") turns $\mathrm{ON}$ in synchronization with the even (respectively, odd) clock phase. Consequently, the capacitor $C_{1}$ charges to a voltage $y_{n}$ during any odd phase, and then delivers all the stored charge to the capacitor $C$ during the next even phase, as a result of the virtual short circuit property of the op-amp. It follows, from the charge conservation principle, that

$$
C\left[x_{n+1}-x_{n}\right]=C_{1} y_{n}
$$

and hence

$$
x_{n+1}=x_{n}+B g\left(x_{n}\right)
$$

which is equivalent to (1) upon choosing

$$
B g\left(x_{n}\right) \triangleq f\left(x_{n}\right)-x_{n} .
$$

To improve our flexibility for realizing an arbitrarily prescribed nonlinear function $f(\cdot)$, we propose the alternate circuit shown in Fig. 5, where the diamond-shaped symbol denotes a "charge" -controlled source which delivers to the capacitor $C$ an incremental charge $\Delta Q=C V_{a}$ during each even phase, where $V_{a} \triangleq V+A x_{n}$ is the controlling voltage. The dynamics ${ }^{3}$ of the circuit in Fig. 5 is described by

$$
x_{n+1}=x_{n}(1+A)+B g\left(x_{n}\right)+V
$$

where $A, B$, and $V$ are parameters to be chosen appropriately. Note that $(7 a)$ is identical to (1) upon choosing

$$
B g\left(x_{n}\right) \triangleq-(1+A) x_{n}-V+f\left(x_{n}\right) .
$$

For example, for the logistic map given in (3) we have $A=4 W-1, B=4 W, V=0$, and $g(x)=-x^{2}$.

In general, a voltage-controlled charge source with a summing controlling node can be efficiently realized by the circuit shown in Fig. 6, where the rightmost element denotes a nullator ${ }^{4}[20]$. During the odd phase, the $j$ th capacitor is charged to $V_{j}^{+}-V_{j}^{-}(j=1,2, \cdots, m)$. Then, during the next even phase, the capacitors are grounded by the nullator and the total charge

$$
\Delta Q=\sum_{j=1}^{m} \Delta Q_{j}=\sum_{j=1}^{m} A_{j} C\left(V_{i}^{+}-V_{j}^{-}\right)
$$

must be injected into the output node. Although a nullator is not available as an isolated physical component, the virtual ground property of the op-amp can be used effectively to provide an equivalent realization. Indeed, in what follows we only need to connect the portion of the circuit of Fig. 6 to the left of the nullator directly to the op-amp via one of the two configurations shown in Fig. 7. Note that the output voltage $V_{o}$ in Fig. 7 at the $n$th time instance is given by

$$
V_{o_{n}}=\sum_{i=1}^{m} A_{i} C\left\{\left(V_{i n-12}^{+}\right)-\left(V_{i n-1 / 2}^{-}\right)\right\}+V_{o n-1}
$$

\footnotetext{
${ }^{3}$ Capacitor $C_{1}$ charges to a voltage $g\left(x_{n}\right)$ during any odd phase, then delivering all the stored charge during the next even phase. Thus the total amount of charge delivered to the capacitor $C$ du ring the even phase is $\Delta Q_{T}=C\left[V+A x_{n}+B g\left(x_{n}\right)\right]$.

${ }^{4} \mathrm{Here}$, it is used for modeling the input port of an op-amp.
} 


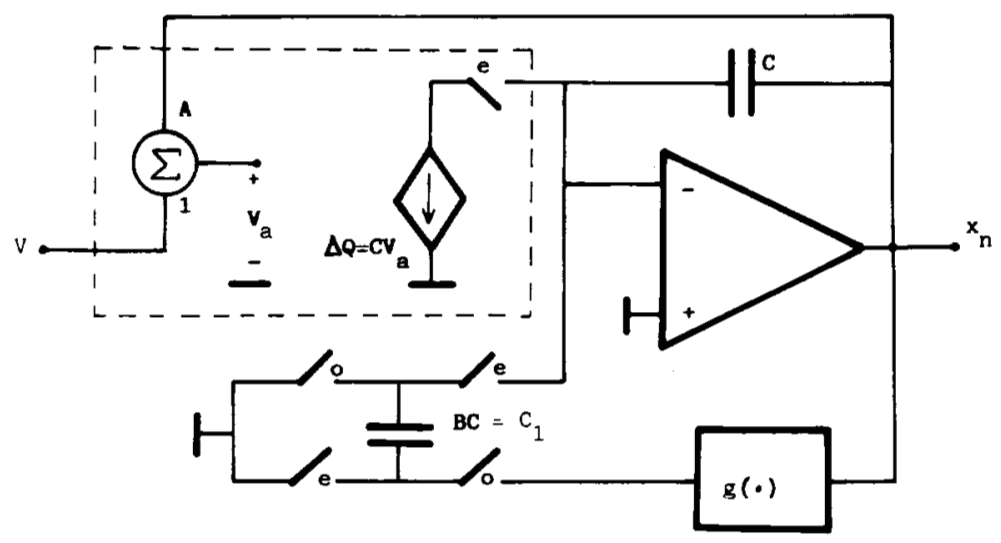

Fig. 5. Conceptual circuit diagram for synthesizing one-dimensional discrete maps using SC circuits.

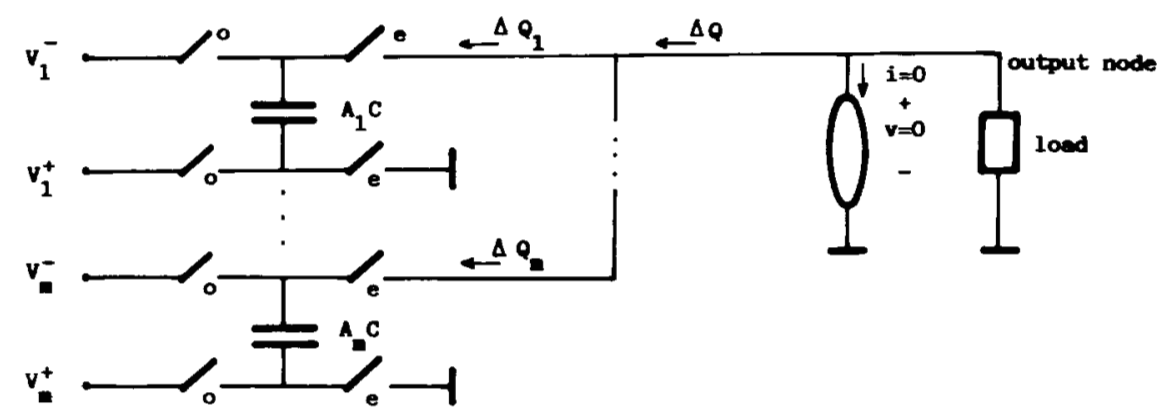

Fig. 6. Grounded-output voltage-controlled summing charge source.
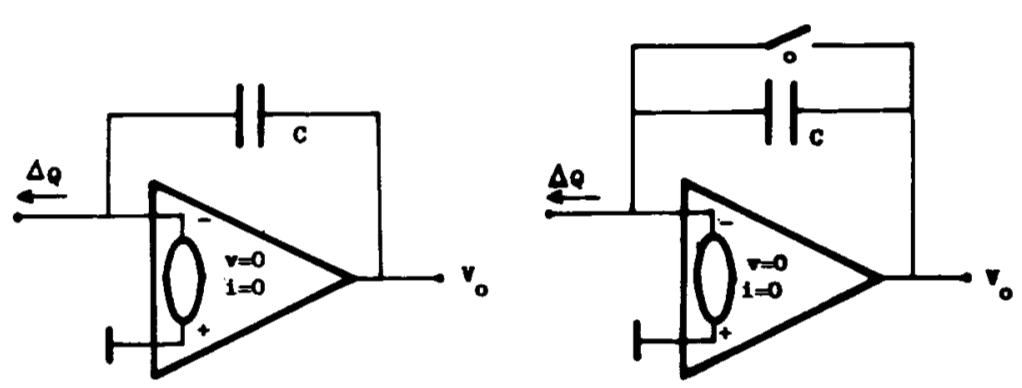

Fig. 7. SC circuit realization for the nullator charge-summing node.

for the circuit on the left and by

$$
V_{o_{n}}=\sum_{j=1}^{m} A_{j} C\left\{\left(V_{i n-12}^{+}\right)-\left(V_{i_{n-12}}^{-}\right)\right\}
$$

for the circuit on the right.

As an example illustrating the above general realization approach, Fig. 8 depicts one possible implementation for the logistic map using the circuit structure of Fig. 5.

\section{B. Circuits for Synthesizing the Nonlinear Operator $f(\cdot)$}

Our ability to use the structure in Fig. 5 for realizing any nonlinear discrete map depends on the availability of a general procedure for synthesizing nonlinear functions. This basic synthesis problem has been extensively studied using both continuous-time as well as switched-capacitor circuits [15]-[19], [21]-[23]. The basic approach for synthesizing an arbitrary nonlinear operator $f(\cdot)$ is to first approximate it in

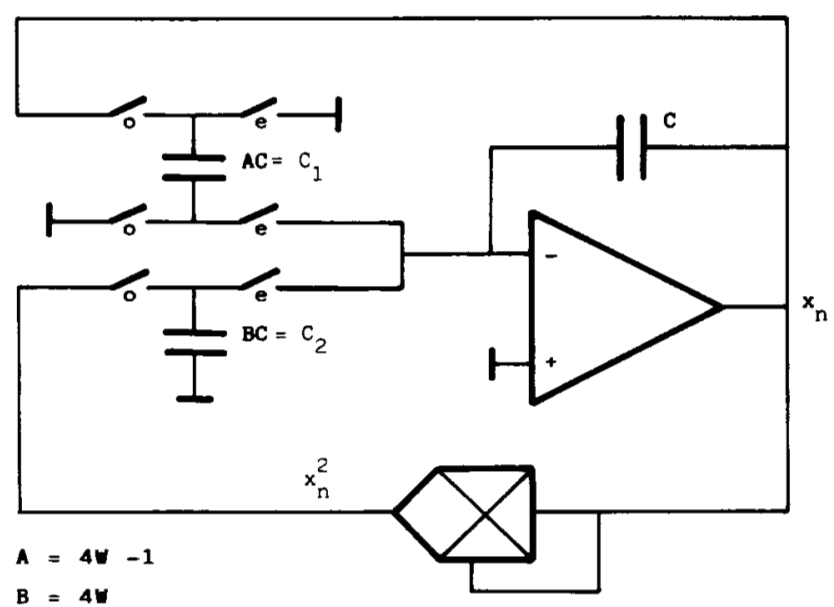

Fig. 8. SC circuit realization of the logistic map using offthe-shelf components. 
terms of some basic set of functions [21], [24] which can be easily synthesized via electronic circuits. One such basic set consisting of multiplication, squaring, modulus, and addition operators has been shown to be easily realized using switched-capacitor circuit techniques [14]-[19]. This basic set motivates us to approximate any nonlinear operator via Piecewise Linear functions or Piecewise Polynomial functions. Moreover, many nonlinear maps reported in the literature are described by either piecewise-linear or piecewise-polynomial functions. Typical examples include the logistic map [8], the Hénon map [25], and the Lozi map [26], [27]. These examples are special cases of the general family of piecewise-linear functions represented by

$$
H_{L}(x)=A x+B+\frac{1}{2} \sum_{j=1}^{N}\left[1+\operatorname{sgn}\left(x-E_{j}\right)\right] M_{i}\left(x-E_{j}\right)
$$

or the general family of piecewise-polynomial functions, represented by

$$
\begin{aligned}
H_{p}(x)= & \sum_{k=0}^{3} A_{k} x^{k}+\frac{1}{2} \sum_{j=1}^{N} \\
& \cdot\left[\sum_{k=2}^{3}\left[1+\operatorname{sgn}\left(x-E_{j}\right)\right] M_{k j}\left(x-E_{j}\right)^{k}\right]
\end{aligned}
$$

where

$$
\operatorname{sgn}\left(x-E_{j}\right)=\left\{\begin{aligned}
1, & x>E_{j} \\
-1, & x \leq E_{j}
\end{aligned}\right.
$$

and $A, B, M_{i}, A_{k}, M_{k j}$, and $E_{i}$ are parameters
Both (10a) and (10b) can be realized using switched-capacitor components. The synthesis of the piecewise-linear function (10a) reduces to a summation of one or more of the following three building blocks:

$$
\begin{aligned}
& H=B \\
& H=A x \\
& H=M(x-E)[1+\operatorname{sgn}(x-E)] .
\end{aligned}
$$

The first two building blocks (11a) and (11b) can be realized by simple linear op-amp circuits. The third building block (11c) can be realized via the switched-capacitor circuit shown in Fig. 9(a). Here the switch labeled $S$ closes during the odd phase only in the case where the value of $x$ is greater than $E$; otherwise, it remains open. This implies that the charge transferred to the capacitor $C$ via the virtual ground of the op-amp is threshold-controlled [17], and can be used to realize the nonlinear term in (11c). The variables $V_{1}$ and $V_{2}$ in Fig. 9(a) depend on the sign of $M$. For $M>0$, we choose $V_{1}=E, V_{2}=x$, and the overall characteristic of the circuit is that shown in Fig. $9(\mathrm{~b})$. On the other hand, if $M<0$, we must choose $V_{1}=x$ and $V_{2}=E$, in order to obtain the characteristics in Fig. 9(c).

The synthesis of the piecewise-polynomial function (10b) reduces to a summation of one or more of the following two building blocks:

$$
\begin{array}{ll}
H=A x^{k}, & k=0,1,2,3 \\
H=M(x-E)^{k}[1+\operatorname{sgn}(x-E)], & k=2,3 .
\end{array}
$$

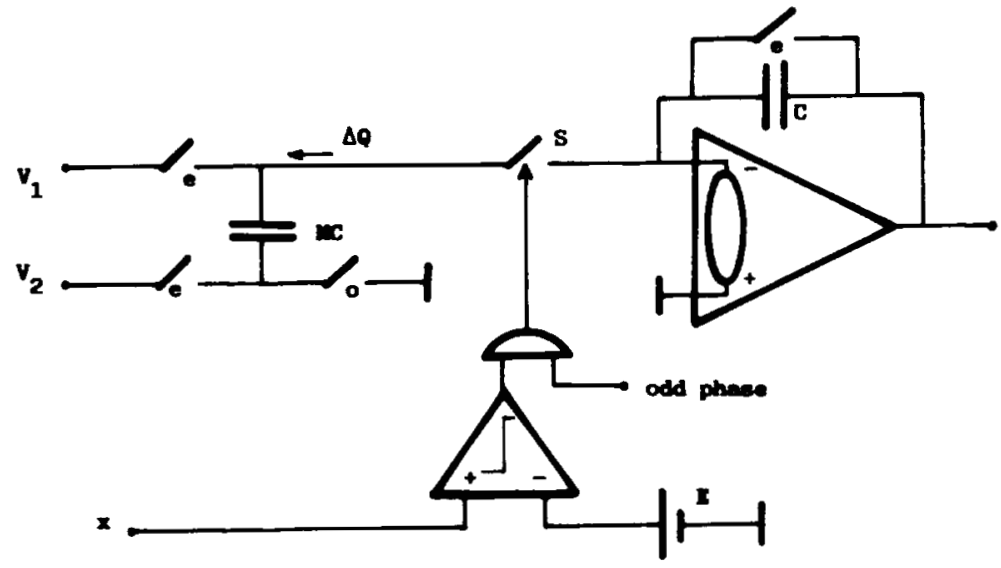

(a)

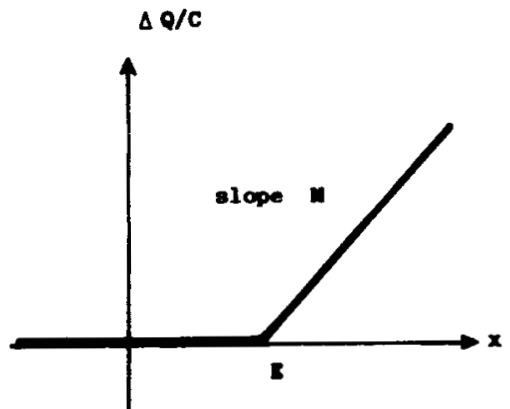

(b)

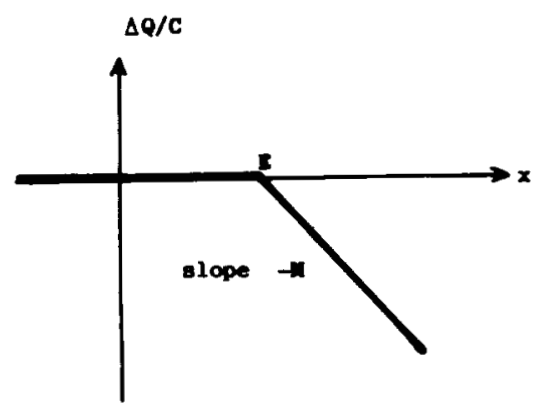

(c)

Fig. 9. (a) An SC basic building block for piecewise-linear functions. (b) Transfer characteristic for $V_{1}=E, V_{2}=x$. (c) Transfer characteristic for $V_{1}=x, V_{2}=E$. 
These building blocks can be realized by combining multipliers and threshold switches. For example, (12b) with $k=$ 3 and $M>0$ can be realized by the circuit shown in Fig. 10 . Note that the summer associated with the op-amp OA1 is slightly different from the summing circuits presented earlier in Fig. 6. The charge in both capacitors is initialized during the odd phase by fixing the voltage across $C_{1}$ and $C_{2}$ to $E$ and 0 volts, respectively. During the even phase, the voltage across $C_{1}$ is set at $x$ volts, thereby causing a charge proportional to $(x-E)$ to flow out of this capacitor. This charge flows into $C_{2}$ and, as a consequence, a voltage equal to $-(x-E)$ appears at the output of OA1. Notice further the output voltage is obtained during the same phase where the signal $x$ is applied. The summer associated with the opamp OA1 is used therefore to avoid an extra half period delay, which could force us to use a more complex phasing scheme for the clock [15].

\section{Extension to Multidimensional Maps}

The circuits and techniques described above can be easily adapted for implementing both two-dimensional and multidimensional maps. Fig. 11 shows the conceptual SC circuit for an m-dimensional map. Applying the charge-conservation principle to this circuit we obtain

$$
x_{j_{n+1}}=v_{j}+g_{j}\left(x_{1_{n}}, x_{2_{n}}, \cdots, x_{m_{n}}\right), \quad j=1,2, \cdots, m .
$$

Equation (13a) is equivalent to (2) upon defining

$$
f_{j}(\cdot) \triangleq v_{j}+g_{j}(\cdot)
$$

The nonlinear multidimensional voltage-controlled charge sources in Fig. 11 can be realized by the same techniques presented in the preceding section. For example, Fig. 12 with $V=1 \mathrm{~V}$ gives a circuit for implementing the Hénon map (5) for $A>0$ and $B>0$.

\section{Experimental Results}

To illustrate the performance of the circuit realization techniques presented in the preceding section, we will present here the experimental results taken from four switchedcapacitor circuits designed for implementing the following four well-known discrete maps; namely, the one-dimensional parabolic map, the one-dimensional piecewise-linear map, the two-dimensional Hénon map, and the twodimensional Lozi map, respectively.

\section{A. Parabolic Map}

The logistic map given in (3) represents the "simplest" example of a parabolic map. It can be realized by the switched-capacitor circuit given in Fig. 8. To adjust the parameter $W$, it is necessary to tune the ratio of the two capacitances $C_{1}$ and $C_{2}$. To simplify the tuning procedure, electronic tuning via the array of binary-weighted capacitors [13] shown in Fig. 13 can be used. The total capacitance seen across terminals (1)-(2) is given by

$$
C_{T}=C \sum_{i=0}^{N} b_{i} 2^{i}
$$

where $b_{i}(i=0,1, \cdots, N)$ denotes a binary digit. We can select a particular value of $C_{T}$ (equivalently, a ratio $C_{T} / C$ ) by

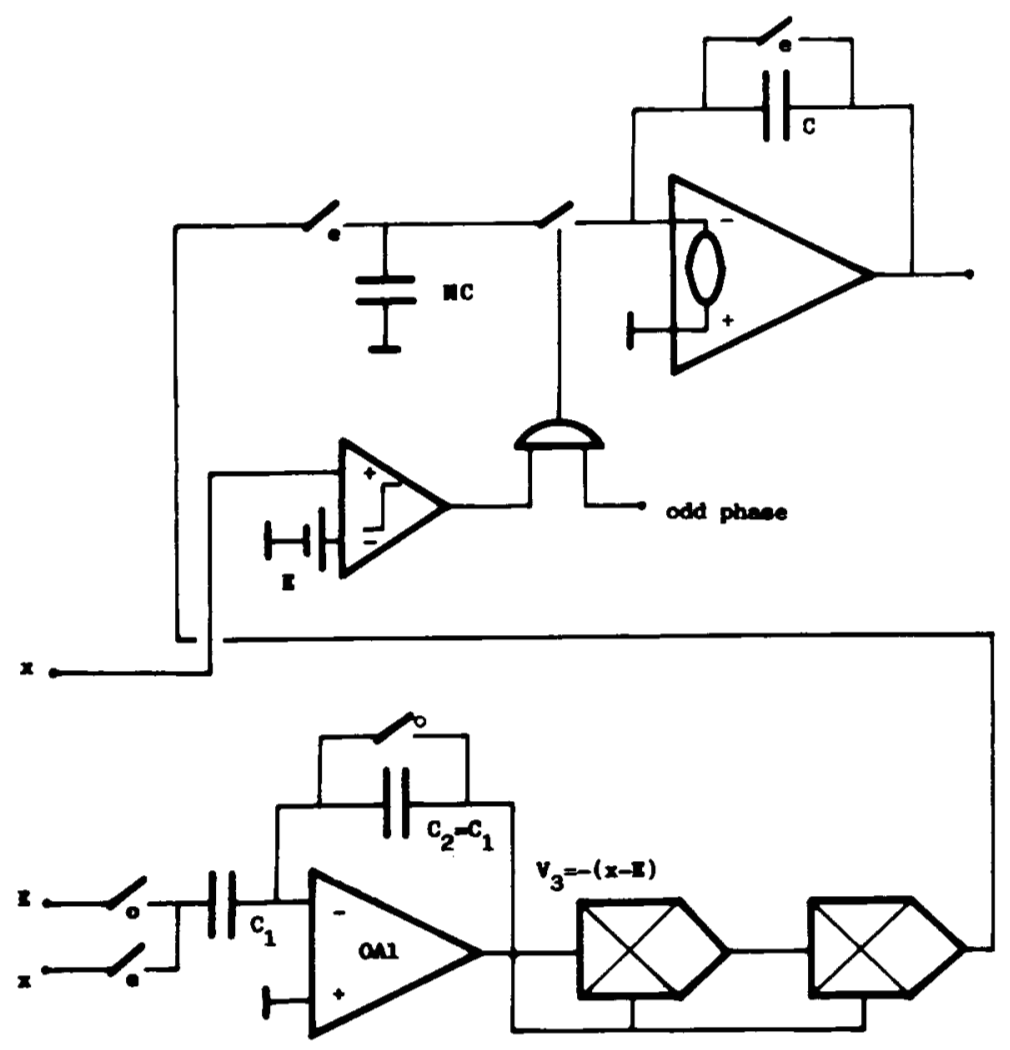

Fig. 10. An SC circuit for realizing a cubic threshold-controlled transfer characteristic. 


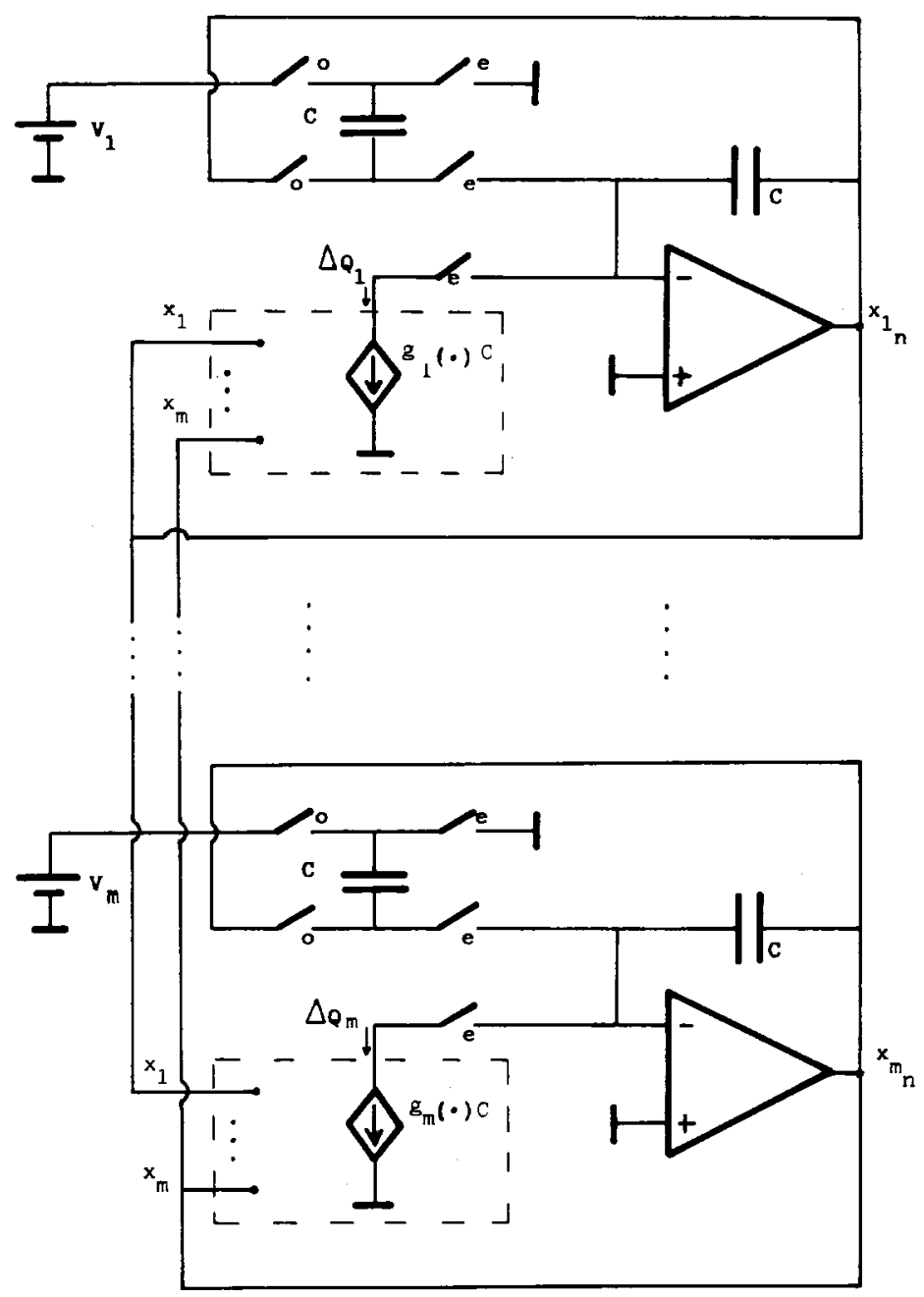

Fig. 11. Conceptual circuit diagram for synthesizing multidimensional maps using SC circuits.

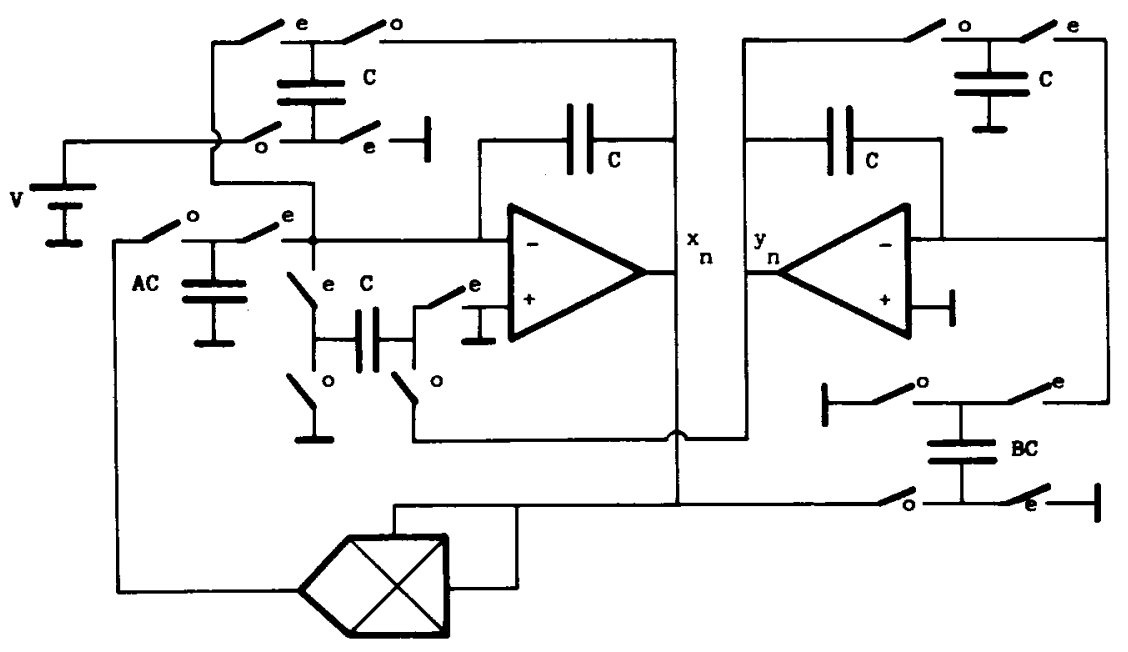

Fig. 12. An SC circuit realization for the Hénon map.

specifying an encoding digital word. Thus a digital word generator can be used to automate the tuning procedure. The additional analog/digital circuitry required in this automatic tuning scheme could be quite costly.
A more economical approach is to realize the parabolic map

$$
x_{n+1}=V-0.5 x_{n}^{2}
$$




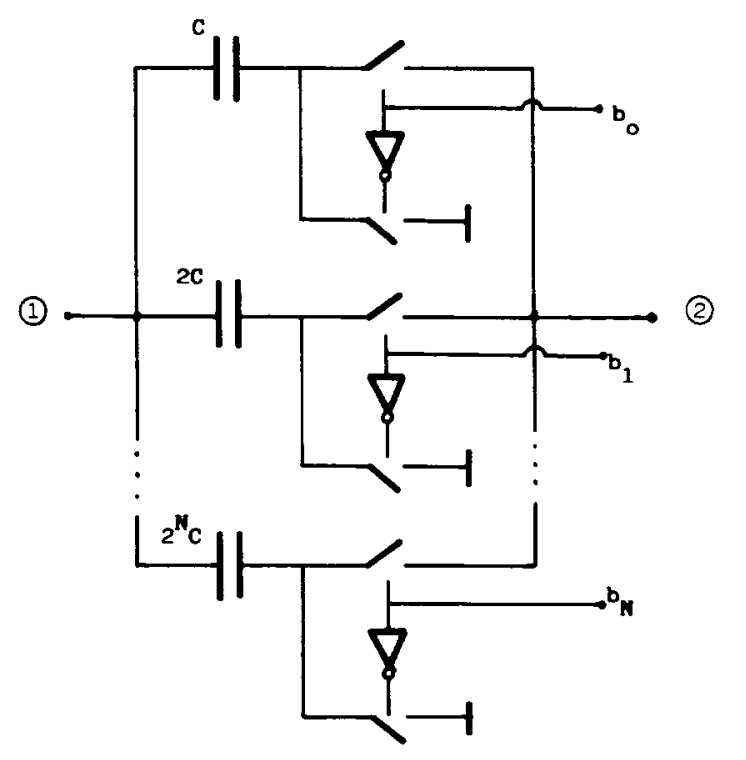

Fig. 13. Digitally controlled binary-weighted capacitor.

which is equivalent to the logistic map via the transformation

$$
z_{n+1}=A x_{n+1}+B
$$

where

$$
W=\frac{1}{8} A \quad B=0.5 \quad A=0.25(-1 \pm \sqrt{1+2 V}) / V .
$$

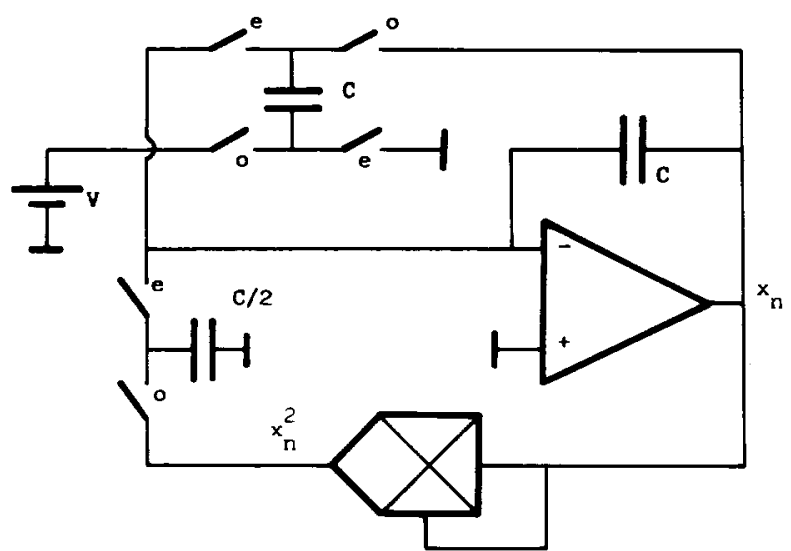

Fig. 14. An SC circuit realization for the parabolic map in (15a).

From the parameter tuning point of view, (15a) is much easier than the logistic map because the parameter $V$ in (15a) can be tuned by a voltage source, rather than the ratio of two capacitances. Fig. 14 shows a switched-capacitor circuit implementation of the map (15a) using our technique. Fig. 15(a) shows the graph representing (15a) for three different values of the parameter $V$. The fixed points are located at the intersections of this family of curves with the straight line in Fig. 15(a). The transformation (15a) maps the interval $[-4,4]$ onto itself where the parameter $V$ is restricted between 0 and 4 . For each value of $V$, the map (15a) has two

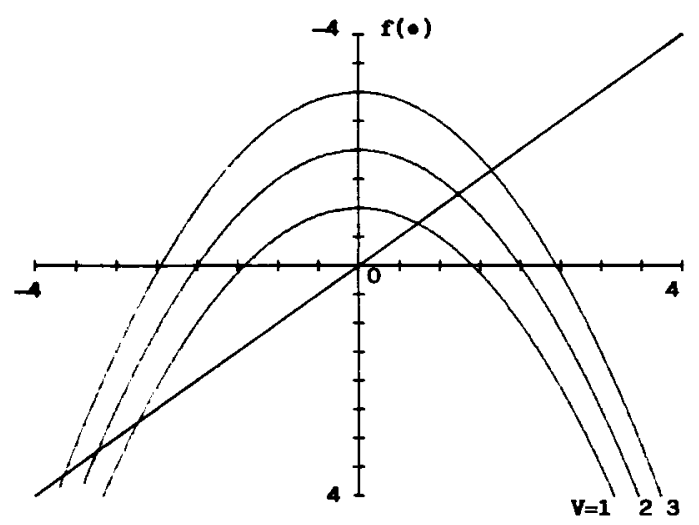

(a)

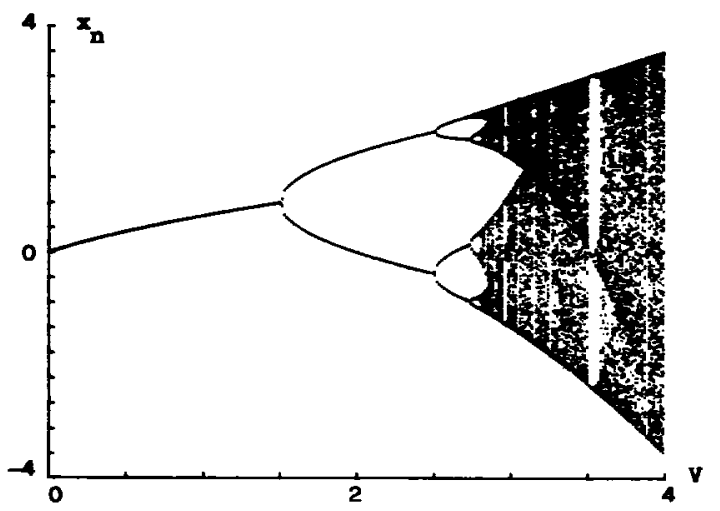

(b)

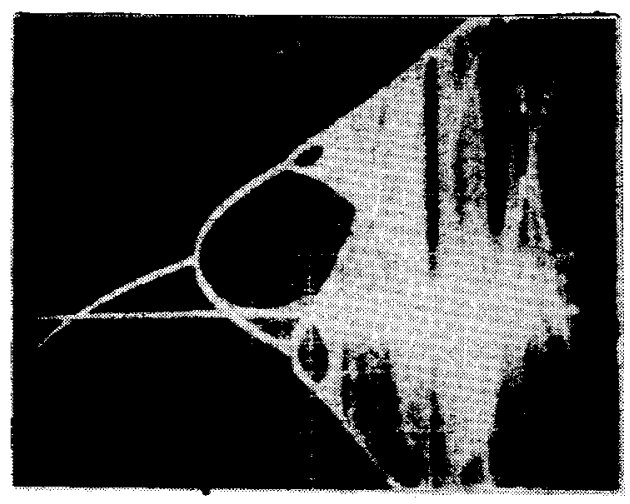

(c)

Fig. 15. (a) Graph of the function defining the map in (15a) for three different values of the parameter $V$. (b) Theoretical bifurcation tree showing the period-doubling route to chaos for the family of maps described by (15a). (c) Measured bifurcation tree by the circuit in Fig. 14 


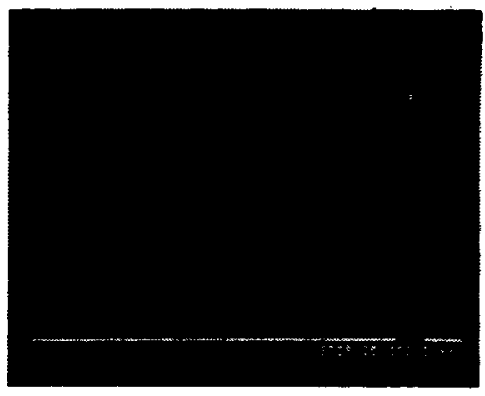

(a)

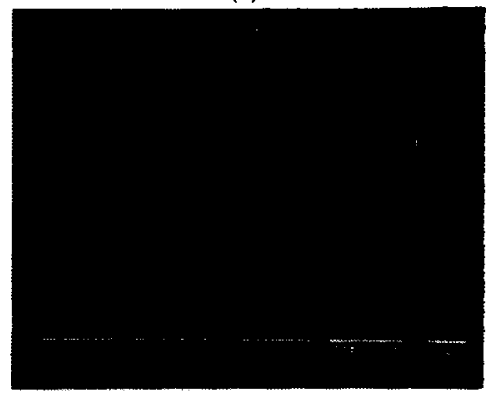

(c)

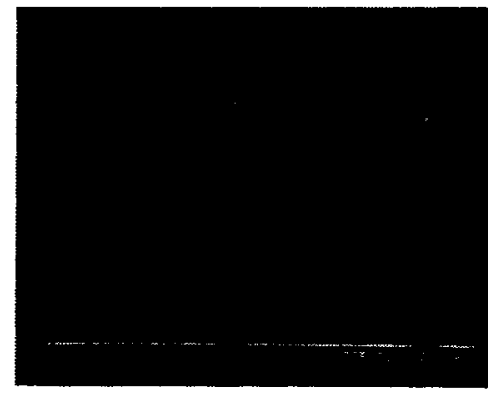

(b)

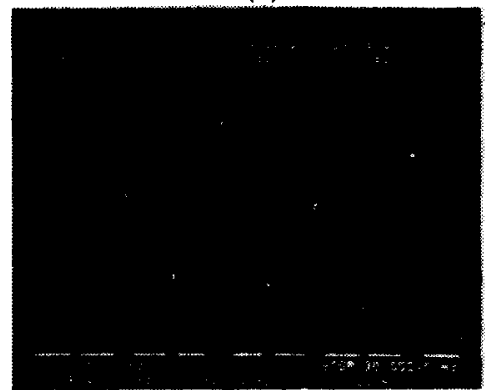

(d)

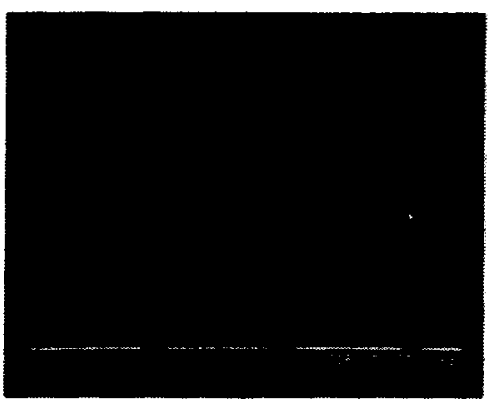

(e)

Fig. 16. Experimental results for the parabolic maps in (15a). (a) Spectra for $V=1 \mathrm{~V}$ (stable fixed point). (b) Spectra for $V=2 \mathrm{~V}$ (period-2 orbit). (c) Spectra for $V=2.6 \mathrm{~V}$ (period-4 orbit). (d) Spectra for $V=2.8 V$ (period-8 orbit). (e) Spectra for $V=3.6 V$ (period-3 orbit).

fixed points located at $x_{1}=-1-\sqrt{1+2 V}$ and at $x_{2}=-1$ $+\sqrt{1+2 V}$. The former is always unstable, while the latter is stable for $V<1.5$. The $\operatorname{map}(15 a)$ has no stable fixed points for $V>1.5$. Fig. 15(b) shows the steady-state accumulation points (i.e., the asymptotic behavior after the transient has decayed to zero) as a function of $V$ (bifurcation tree). This bifurcation tree is an example of a typical route to chaos via period-doubling. The circuit in Fig. 14 was built using $\mu \mathrm{A741}$ operational amplifiers, MC14066 analog switches, AD53KH analog multipliers, and ceramic capacitors with a 20-percent tolerance. Fig. 15(c) shows the bifurcation tree measured from this circuit. The period-doubling route to chaos observed from Fig. 15(c) is further confirmed by the series of spectra associated with the signal $x^{e}\left(x^{e} \triangleq 0\right.$ during any odd phase) shown in Fig. 16(a)-(d). Here, the parameter $V$ is chosen so that the spectra in Fig. 16(a)-(d) correspond to a stable fixed point (Fig. 16(a)), a period-2 orbit (Fig. 16(b)), a period-4 orbit (Fig. 16(c)), and a period-8 orbit (Fig. 16(d)). In addition, existence of a period-3 window in Fig. 14(a) is confirmed by the corresponding spectra shown in Fig. 16(e).

\section{B. Piecewise-Linear Map}

Consider next the piecewise-linear map

$$
x_{n+1}=V-\left(\left|x_{n}\right|-0.4\right)\left[1+\operatorname{sgn}\left(\left|x_{n}\right|-0.4\right)\right]
$$

where $x \in[-4,4]$ for $0 \leq V \leq 4$.
Fig. 17(a) shows the graph of (16) for three different values of $V$. The bifurcation tree for this family of maps is shown in Fig. 17(b). Note that unlike the logistic map where the period-3 window is extremely narrow and difficult to observe, here the period-3 window is rather wide, namely, $2<V<2.8$.

Fig. 18 shows a switched-capacitor circuit implementation $^{5}$ of the family of maps defined by (16). This circuit was built using off-the-shelf components similar to those used in the logistic map, and a $\mu$ A709 analog comparator. Fig. 19(a) shows an oscillogram of the resulting experimental bifurcation diagram. More details of this diagram which shows a period- 3 window and a period -5 window are given in Fig. 19. Their agreement with that predicted by Fig. 17(b) is indeed excellent. Fig. 20(a)-(g) shows nine periodic signals measured from this circuit as $V$ increases from 0 to 4 . Here, the output signal $x^{e}(t)$ appears in the upper trace. The lower trace is the clock signal which remains fixed in all measurements. Note that the nine output signals have a

${ }^{5}$ Note that the input of the nonlinear block is sampled during the even phase, thereby making it unnecessary to hold the signal $x$ during the odd phases. This allows us to simplify the circuit by eliminating the switches $S_{1}, S_{2}$, and the associated capacitor, and substituting them by an odd analog switch shunted across the feedback capacitor of the amplifier OA2. 


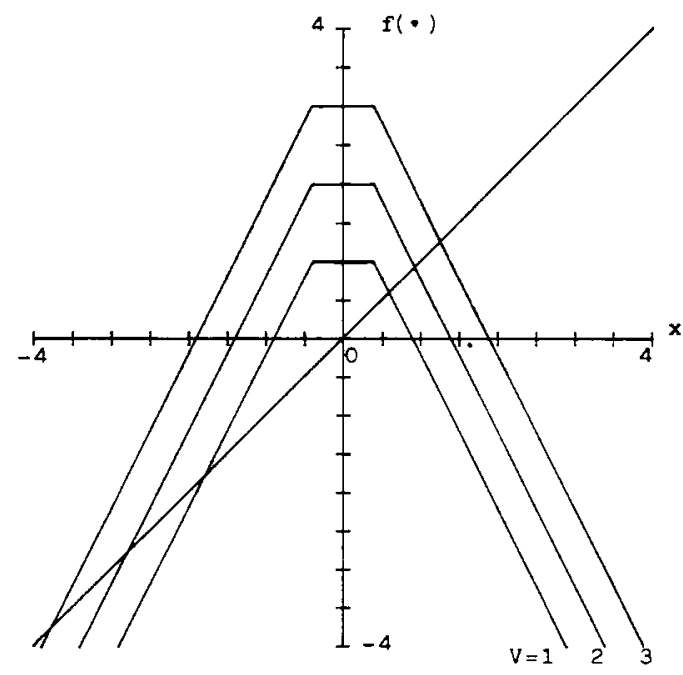

(a)

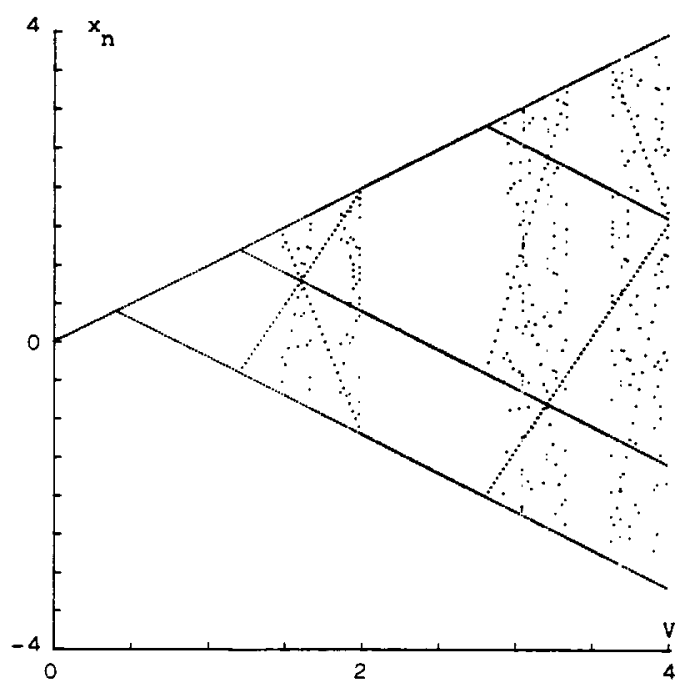

(b)

Fig. 17. (a) Graph of the function for the map in (16) for three different values of $V$. (b) Bifurcation tree for this family of maps.

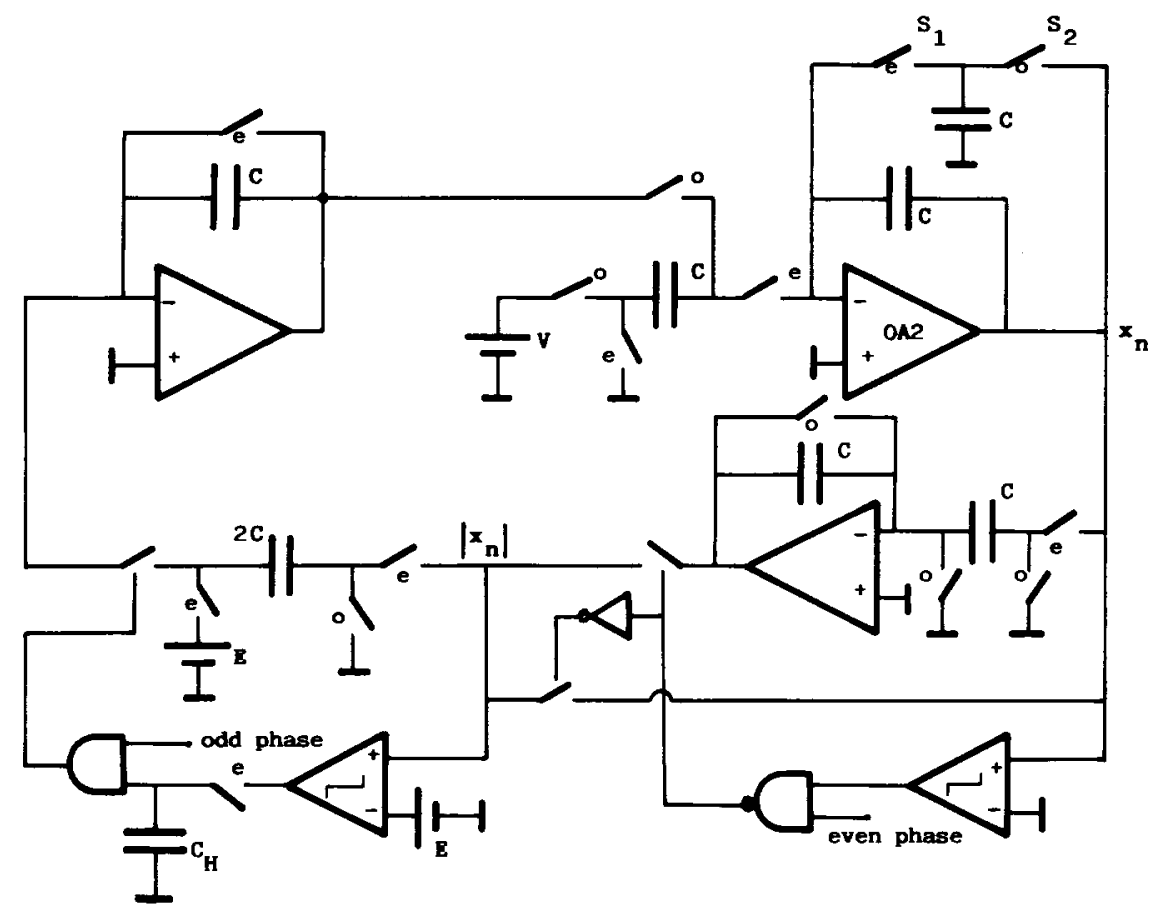

Fig. 18. An SC circuit realization for the piecewise-linear map in (16).

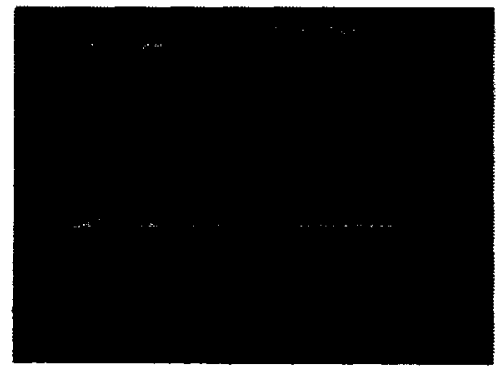

(a)

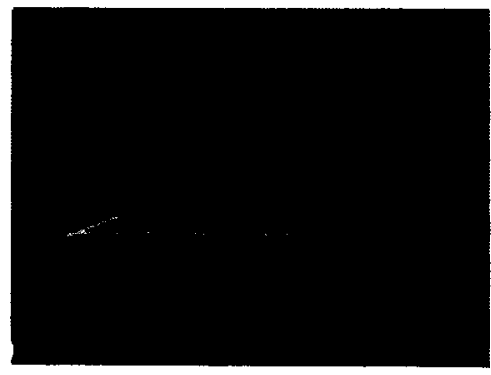

(b)

Fig. 19. (a) Experimental bifurcation diagram for the circuit of Fig. 18. (b) An enlarged view of the same diagram showing odd-period windows. 


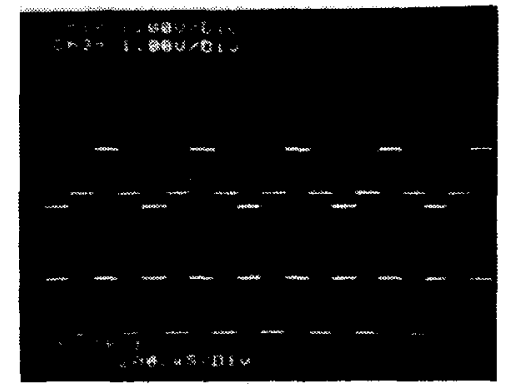

(a)

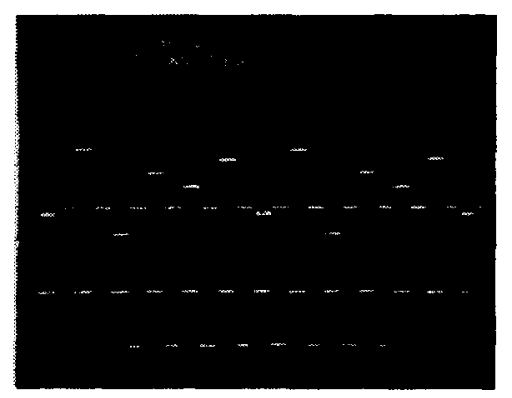

(c)

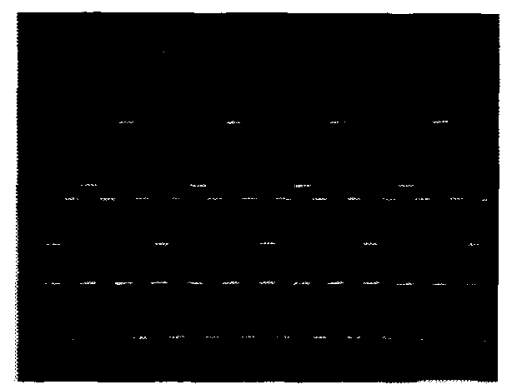

(e)

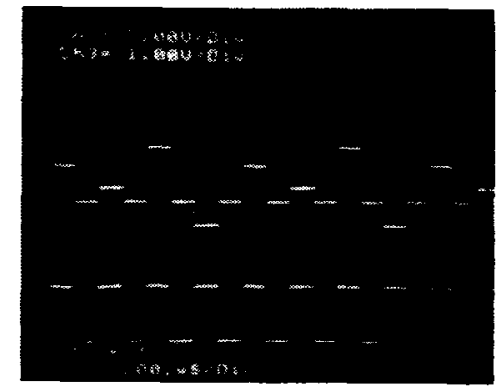

(b)

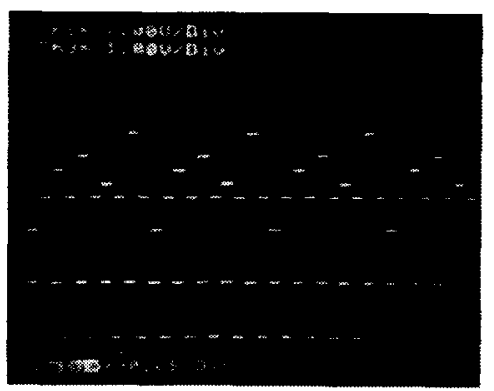

(d)

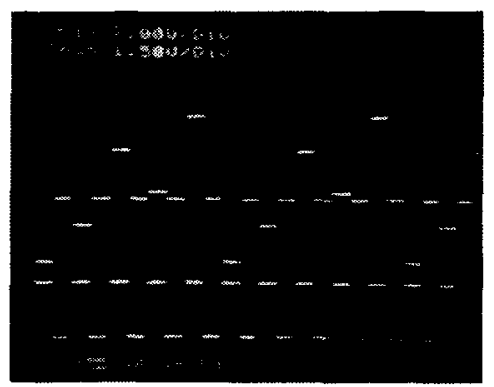

(f)

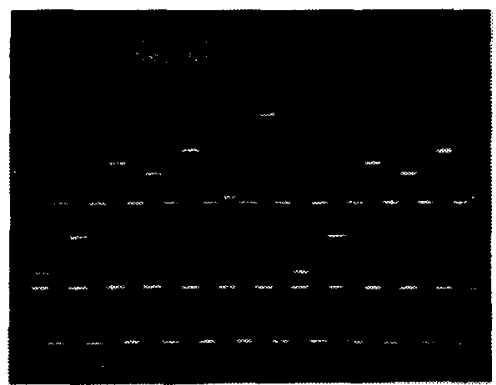

(g)

Fig. 20. Different periodic signals obtained from the circuit of Fig. 18 as the parameter $V$ increases from $0 \mathrm{~V}$. In all of the oscillograms, the lower trace is the clock signal. (a) $V=1.3 V$ (period 2). (b) $V=1.6 \mathrm{~V}$ (period 4). (c) $V=1.66 \mathrm{~V}$ (period 6). (d) $V=1.86 \mathrm{~V}$ (period 5). (e) $V=2.24 \vee$ (period 3). (f) $V=3.62 \vee($ period 5). (g) $V=3.86 \vee($ period 7$)$.

period (relative to the clock signal) equal to $2,4,6,5,3,5$, and 7 , respectively. Finally, Fig. 21(a)-(c) shows the frequency spectra for three cases where the measured output signal has an odd periodicity; namely, 3, 5, and 7.

\section{Hénon Map}

Consider next the two-dimensional map

$$
\begin{aligned}
& x_{n+1}=V-K x_{n}^{2}+y_{n} \\
& y_{n+1}=K^{\prime} x_{n}
\end{aligned}
$$

which is equivalent to the Hénon map defined in (5) via the transformation

$$
\begin{aligned}
& x_{n+1}=V z_{n+1} \\
& y_{n+1}=V w_{n+1}
\end{aligned}
$$

and assuming $K^{\prime}=B$ and $K=A W$.

The advantage of using (17a) over the equivalent Hénon map is that we can use a voltage source, $V$, as a controlling parameter. For convenience, both $K$ and $K^{\prime}$ are assumed to be equal to 0.5 . In Fig. 22(a) we show a computer-generated 


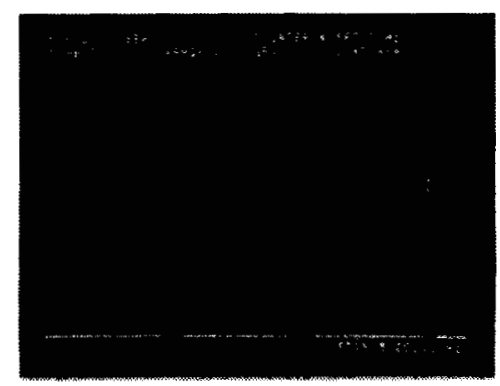

(a)

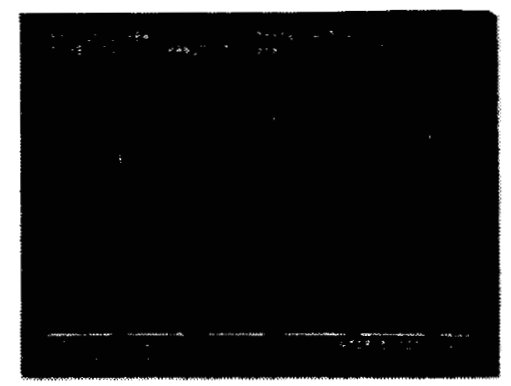

(b)

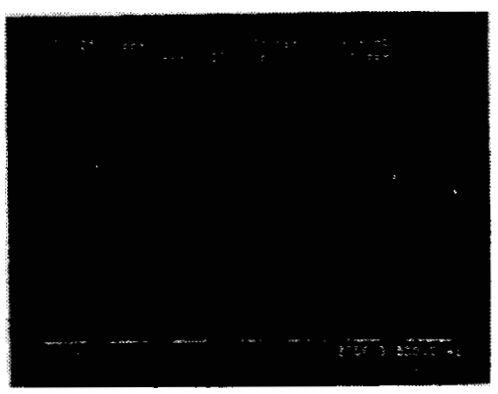

(c)

Fig. 21. Frequency spectra for some odd-period output signals for the circuit of Fig. 18. (a) $V=2.24 V$ (period 3). (b) $V=1.86 V$ (period 5). (c) $V=3.86 V$ (period 7 ).

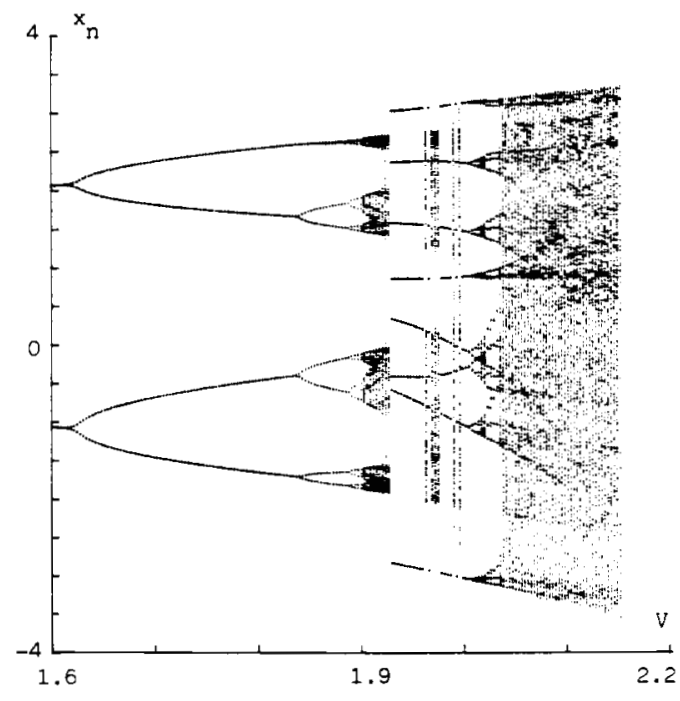

(a)

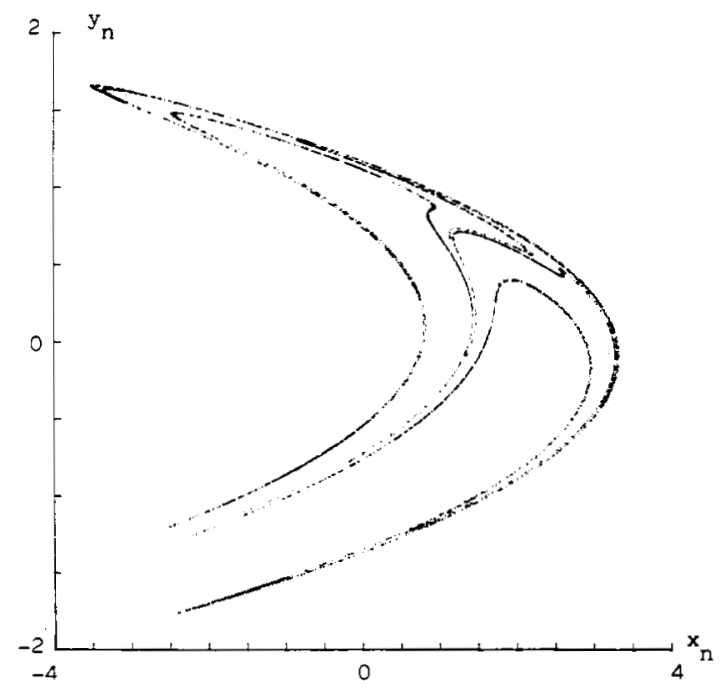

(b)

Fig. 22. Computer simulations of the map described by (17a). (a) Detail of the bifurcation tree for the variable $x$. (b) The strange attractor.

bifurcation tree for the variable $x$ as $V$ varies from 1.6 to 2.2 V. A two-dimensional plot showing a strange attractor obtained with $V=2.14 \mathrm{~V}$ is shown in Fig. 22(b).

We have carried out many experimental measurements associated with (17a) using the circuit structure of Fig. 12. Fig. 23(a) shows an oscillogram corresponding to the attractor for the actual circuit with $V=2.16 \mathrm{~V}$. Additional oscillograms corresponding to other values of $V$ are shown in
Fig. 23(b)-(f). Note that the attractor in Fig. 23(a) bifurcates into several isolated islets as the parameter $V$ is reduced slowly from $2.16 \mathrm{~V}$. The theoretical and experimental results are found to be in a good agreement (the largest error in the parameter value was found to be 2 percent). It is interesting to note that due to component tolerances, the physical circuit in Fig. 12 cannot be exactly modeled by (17b). Nevertheless, the fact that the chaotic attractor measured 


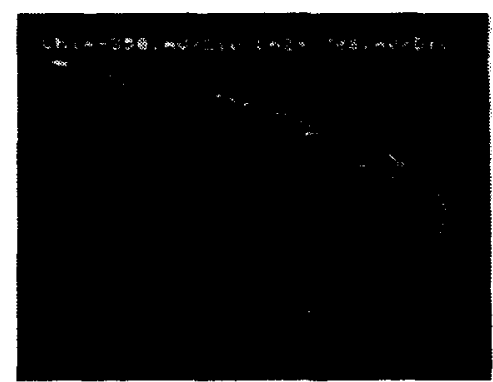

(a)

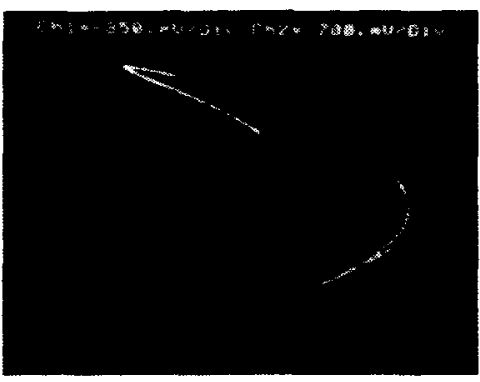

(c)

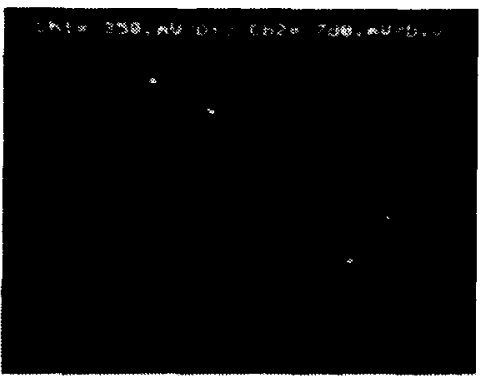

(e)

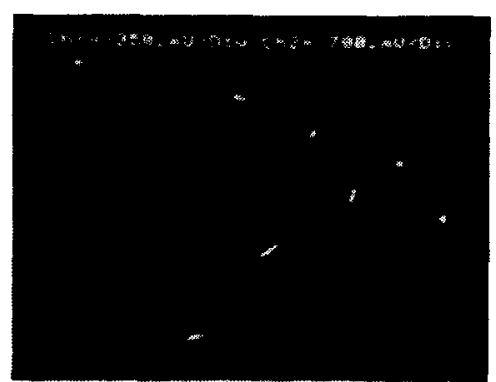

(b)

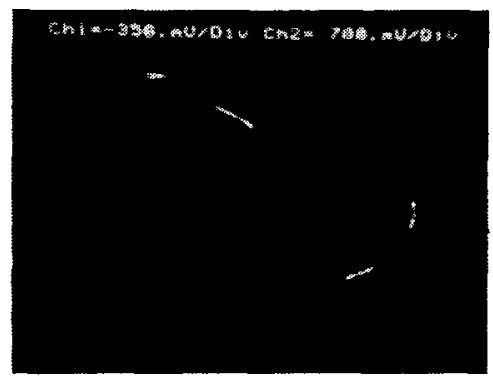

(d)

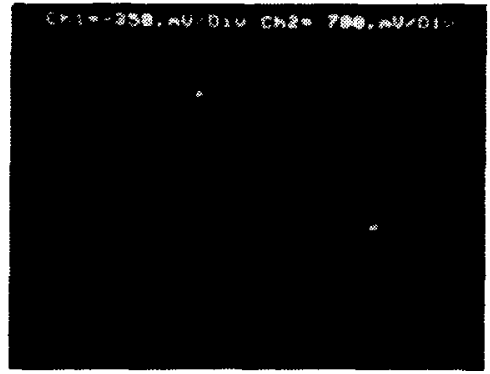

(f)

Fig. 23. Oscilloscope pictures showing several interesting attractors displayed by the circuit in Fig. 12. (a) $V=2.16 \vee$ (the experimental attractor), (b) $V=2.05 \vee$ (8 islets). (c) $V=$ $2 \mathrm{~V}$ ( 2 islets). (d) $V=1.91 \mathrm{~V}$ (4 islets). (e) $V=1.76 \mathrm{~V}$ (period 4). (f) $V=1.48 \mathrm{~V}$ (period 2).

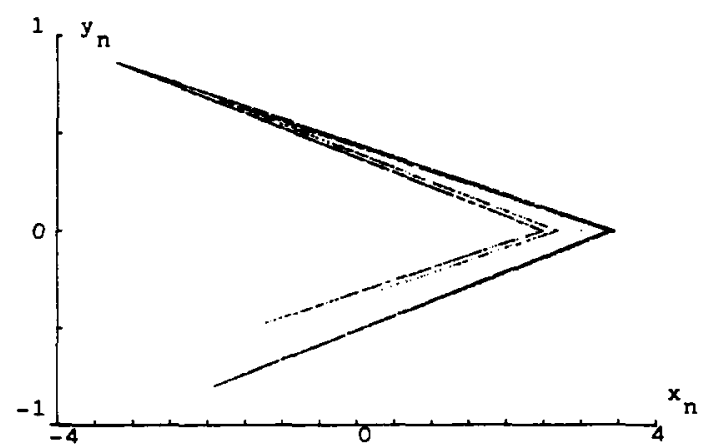

(a)

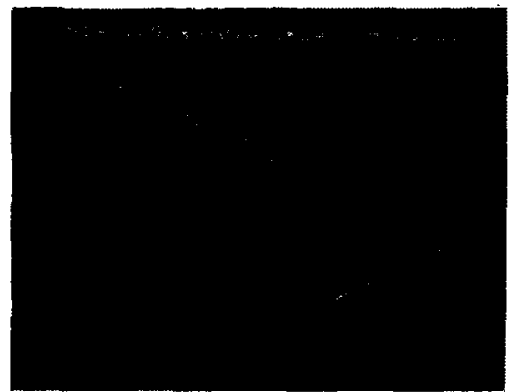

(b)

Fig. 24. (a) Computer simulation of the strange attractor for the map described by (18).

(b) Oscilloscope picture showing the actual attractor displayed by the circuit in Fig. 25.

from this circuit is remarkably similar to the theoretically calculated Hénon's attractor shows the robustness of our circuit model.

\section{Lozi Map}

For our final example, consider the scaled version of the well-known two-dimensional piecewise-linear Lozi map
[26], [27] given by

$$
\begin{aligned}
& x_{n+1}=3+y_{n}-A\left|x_{n}\right| \\
& y_{n+1}=B x_{n} .
\end{aligned}
$$

A computer-generated attractor for (18) with $A=1.8$ and $B=0.25$ is shown in Fig. 24(a). A switched-capacitor circuit realization of this two-dimensional map is shown in Fig. 25. 


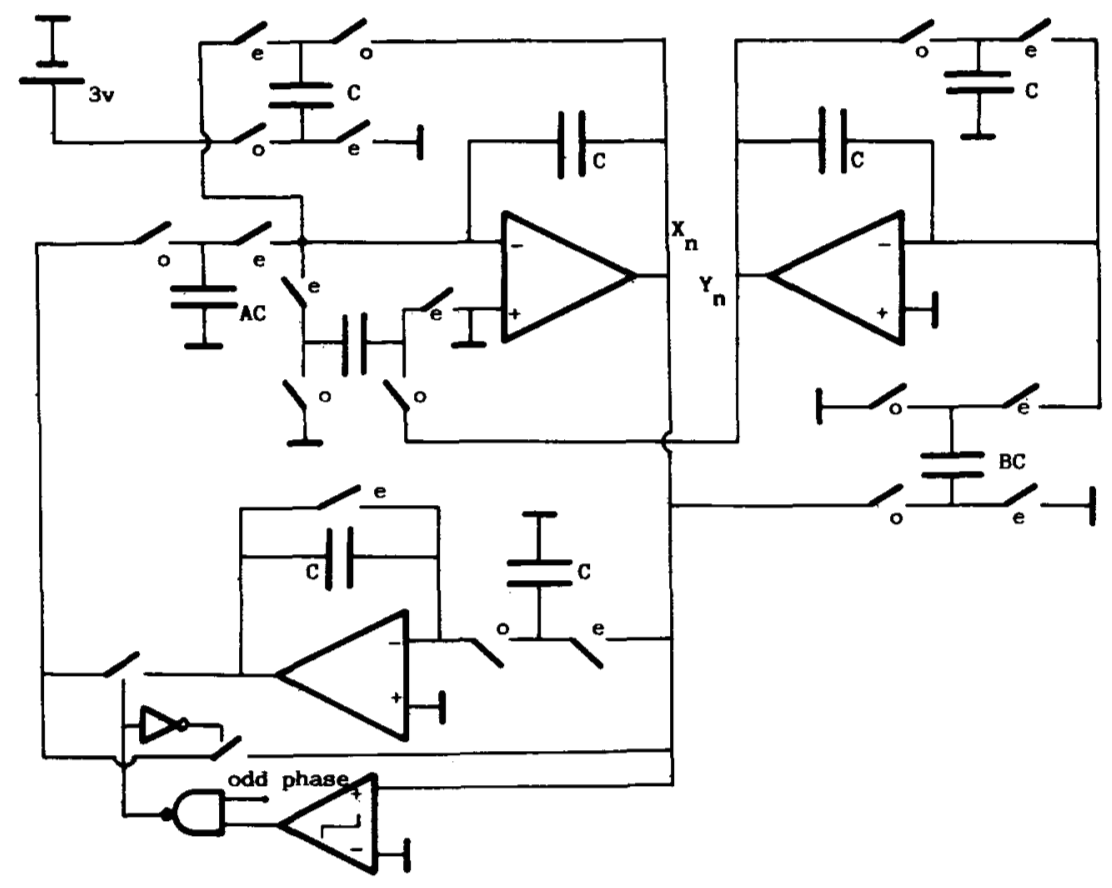

Fig. 25. SC circuit realization for the map in (18).

The corresponding attractor measured from this circuit is shown in Fig. 24(b). Again, note the excellent agreement between the theoretical and experimental results shown in Fig. 24(a) and (b), respectively.

\section{REFERENCES}

[1] H. G. Schuster, Deterministic Chaos: An Introduction. Weinheim, West Germany: Physik-Verlag, 1984.

[2] H. Bai-Lin, Chaos. Singapore: World Scientific, 1984.

[3] P. Collet and J. P. Eckmann, Iterated Maps on The Interval as Dynamic Systems. Boston, MA: Birkhauser, 1980.

[4] R. L. Devaney, An Introduction to Chaotic Dynamical Systems. Menlo Park, CA: Benjamin-Cummings Publ., 1986.

[5] A. I. Mees and C. T. Sparrow: Chaos, Proc. Inst. Elec. Eng., vol. 128, pt. D, pp. 201-205, Sept. 1981.

[6] L. O. Chua and P. M. Lin, Computer-Aided Analysis of Electronic Circuits: Algorithms and Computational Techniques. Englewood Cliffs, NJ: Prentice-Hali, 1975.

[7] J. L. Huertas, A. Rueda, B. Perez-Verdu, and A. Rodriguez-Vazquez, "Computer simulation of chaotic networks: Limitations of circuit-level simulators," in Proc. Int. Conf. on Computers Systems and Signal Processing (Bangalore, India, Dec. 1984).

[8] R. M. May, "Simple mathematical models with very complicated dynamics," Nature, vol. 261, pp. 459-467, June 1976.

[9] M. J. Feigenbaum, "Quantitative universality for a class of nonlinear transformations," J. Statist. Phys., vol. 19, no. 1, pp. 25-52, 1978.

[10] - "Universal behavior in nonlinear systems," Los Alamos Sci., pp. 4-27, Summer 1980.

[11] I. Gumowski and C. Mira: Recurrences and Discrete Dynamic Systems. Berlin-Heidelberg, West Germany: Springer-Verlag, 1980.

[12] A. Rodriguez-Vazquez, J. L. Huertas, and L. O. Chua, "Chaos in a switched-capacitor circuit," IEEE Trans. Circuits Syst., vol. CAS-32, pp. 1083-1085, Oct. 1985.

[13] R. Gregorian, K. W. Martin, and G. C. Temes, "Switchedcapacitor circuit design," Proc. IEEE (Special Section on Switched-Capacitor Circuits), vol. 71, pp. 941-966, Aug., 1983.

[14] P. E. Allen and E. Sanchez-Sinencio, Switched-Capacitor Circuits. New York, NY: Van Nostrand Reinhold, 1984.

[15] A. Rodriguez-Vazquez, "Nonlinear switched-capacitor cir- cuits," Ph.D. dissertation, U. Sevilla, Seville, Spain, 1982 (in Spanish).

[16] J.L. Huertas, A. Rodriguez-Vazquez, and A. Rueda, "Low-order polynomial curve fitting using switched-capacitor circuits," in Proc. 1984 Int. Symp. on Circuits and Systems (Montreal, Que, Canada, May 1984), pp. 1123-1126.

[17] J. L. Huertas, L. O. Chua, A. Rodriguez-Vazquez, and A. Rueda: "Nonlinear switched-capacitor networks: Basic principles and piecewise-linear design," IEEE Trans. Circuits Syst., vol. CAS32, pp. 305-319, Apr. 1985.

[18] A. Rodriguez-Vazquez, I. L. Huertas, and L. O. Chua, "On a class of $\mathrm{SC}$ resistors and its application to the synthesis of nonlinear driving-point and transfer characteristic plots," Int. J. Circuit Theory Appl., vol. 13, pp. 309-326, Oct. 1985.

[19] J. L. Huertas and A. Rodriguez-Vazquez, "Rational switchedcapacitor networks," in Proc. 1986 Int. Symp. on Circuits and Systems (San Jose, CA, May 1986).

[20] L. T. Bruton, RC-Active Circuits. Englewood Cliffs, NJ: Prentice-Hall, 1980.

[21] J. L. Huertas and A. Rueda, "Sectionwise piecewise polynomial functions: Application to the analysis and synthesis of nonlinear n-port networks," IEEE Trans. Circuits Syst., vol. CAS-31, pp. 897-905, Oct. 1984.

[22] A. Rueda, J. L. Huertas, and A. Rodriguez-Vazquez, "Basic circuit structures for the synthesis of piecewise polynomial onePort resistors," Proc. Inst. Elec. Eng., vol. 132, pt. G, pp. 123130, Aug. 1985.

[23] L. O. Chua and S. Wong, "Synthesis of piecewise-linear networks," Electron. Circuits Syst., vol. 2, pp. 102-108, July 1978.

[24] L.O. Chua and S. M. Kang, "Sectionwise piecewise-linear networks: Canonical representations, properties and applications," Proc. IEEE, vol. 65, pp. 915-929, June 1977.

[25] M. Hénon, "A two-dimensional mapping with a strange attractor," Commun. Math. Phys., vol. 50, pp. 69-77, 1976.

[26] R. Lozi, "Un attracteur etrange du type attracteur de Hénon," J. Phys. (Paris), vol. 39 (coll. C5), pp. 9-10, 1978 (in French).

[27] M. Misiurewicz, "Strange attractors for the Lozi mappings," Ann. New York Acad. Sci., pp. 348-358, 1980.

[28] J. L. Hudson and O. E. Rossler, "A piecewise-linear invertible noodle map," Physica, vol. 11D, pp. 239-242, 1984.

[29] S. Tanaka, T. Matsumoto, and L. O. Chua, "Bifurcations of a driven R-L-diode circuit," in Proc. 1985 Int. Symp. on Circuits and Systems (Kyoto, Japan, 1985), pp. 851-854.

[30] T. Saito, "A chaos generator based on a quasi-harmonic oscillator," IEEE Trans. Circuits Syst., vol. CAS-32, pp. 320-331, 1985. 


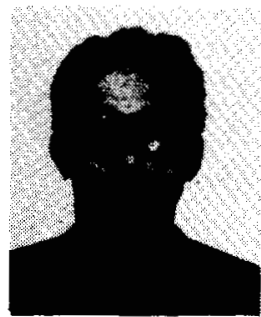

Angel Rodriguez-Vazquez (Member, IEEE) was born in Seville, Spain. He received the Licenciado en Fisica degree in 1977 and the Doctor en Ciencias Fisicas degree in 1983 both from the University of Seville.

Since October 1976, he has been with the Departamento de Electricidad y Electronica at the University of Seville, where he became Assistant Professor in 1978 and Associate Professor in Electronics in 1984. His research interest lies in the fields of ana$\log$ circuit design and nonlinear networks analysis and synthesis.

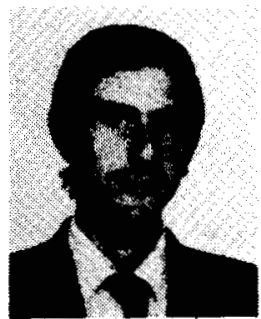

Jose L. Huertas (Member, IEEE) was born in Seville, Spain. He received the Licenciado en Fisica degree in 1969, and the Doctor en Ciencias Fisicas degree in 1973, both from the University of Seville.

From October 1970 to September 1971 he was with the Philips International Institute, Eindhoven, The Netherlands, as a PostGraduate Student. Since October 1971, he has been with the Departamento de Electricidad $y$ Electronica at the University of Seville, where he became Assistant Professor in 1973 and a Professor in Electronics in 1981. His research interest lies in the fields of multivalued logic, sequential machines, analog circuit design, and nonlinear networks analysis and synthesis.

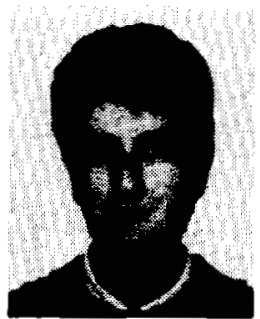

Adoracion Rueda was born in Puertollano, Spain. She received the Licenciado en Fisica degree in 1976, and the Doctor en Ciencias Fisicas degree in 1982, both from the University of Seville, Seville, Spain.

Since October 1975, she has been with the Departamento de Electricidad y Electronica at the University of Seville, where she became Assistant Professor in 1976 and Associate Professor in Electronics in 1984. Her research interest lies in the fields of analog circuit design and nonlinear networks analysis and synthesis.

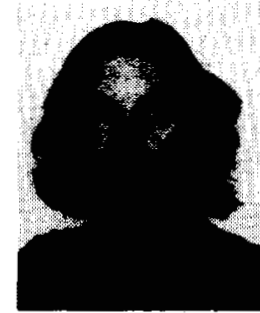

Belen Perez-Verdu was born in Monovar, Spain. She received the Licenciado en Fisica degree in 1979, and the Doctor en Ciencias Fisicas degree in 1985, both from the University of Seville, Seville, Spain.

Since October 1978, she has been with the Departamento de Electricidad y Electronica at the University of Seville, where she is employed as an Assistant Professor. Her research interest lies in the fields of works analysis and synthesis. analog circuit design and nonlinear nẹt-

Leon O. Chua (Fellow, IEEE), for a photograph and biography please see page 980 of this issue. 


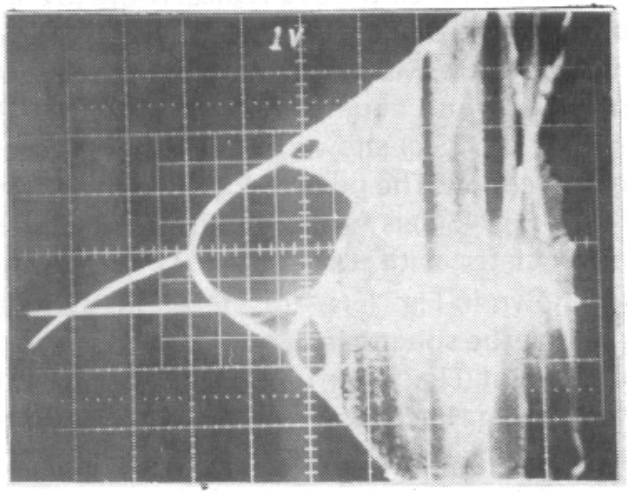

ersidad de Sevilla. Downloaded on)April 20,2020 at 15:51:37 UTC 


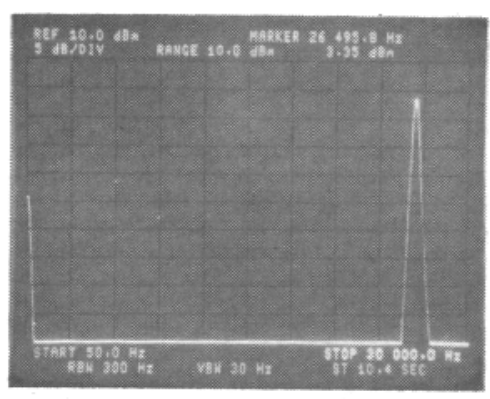

(a)

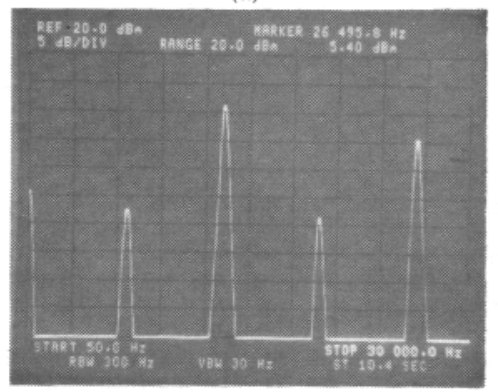

(c)

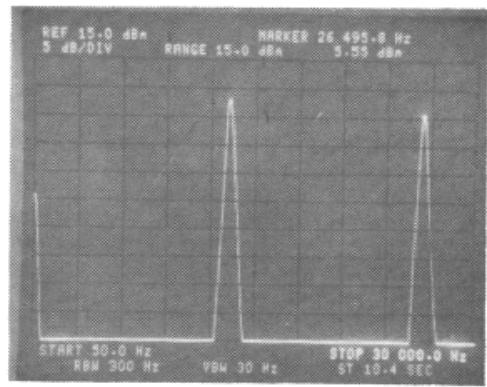

(b)

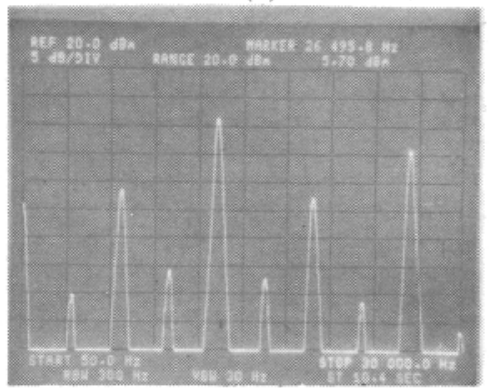

(d)

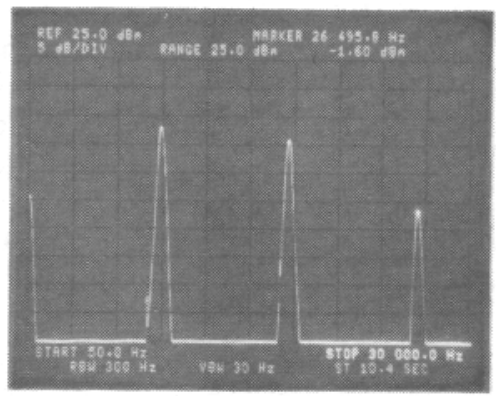

(e)

Fig. 16. Experimental results for the parabolic maps in (15a). (a) Spectra for $V=1 \mathrm{~V}$ (stable fixed point). (b) Spectra for $V=2 \mathrm{~V}$ (period-2 orbit). (c) Spectra for $V=2.6 \mathrm{~V}$ (period-4 orbit).

(d) Spectra for $V=2.8 \mathrm{~V}$ (period-8 orbit). (e) Spectra for $V=3.6 \mathrm{~V}$ (period-3 orbit). uthorized licensed use limited to: Universidad de Sevilla. Downloaded on April 20,2020 at 15:51:37 UTC from IEEE Xplore. Restrictions apply 


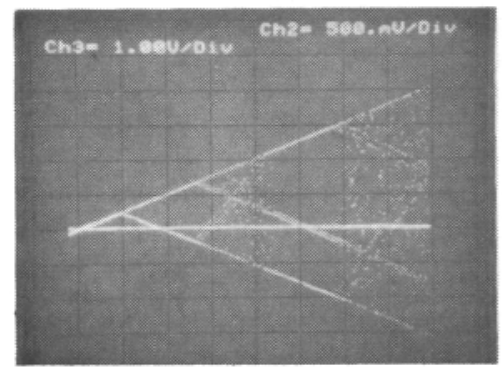

(a)

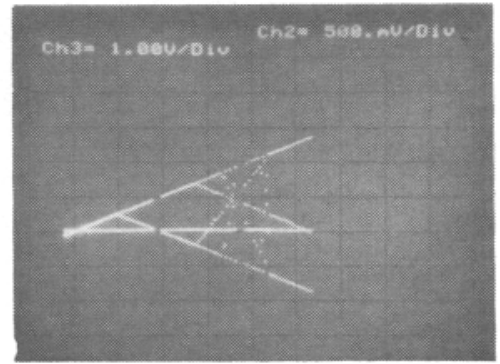

(b)

Fig. 19. (a) Experimental bifurcation diagram for the circuit of Fig. 18. (b) An enlarged view of the same diagram showing odd-period windows. uthorized licensed use limited to: Universidad de Sevilla. Downloaded on April 20,2020 at 15:51:37 UTC from IEEE Xplore. Restrictions apply 


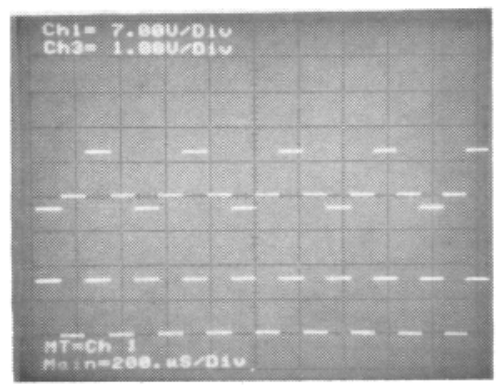

(a)

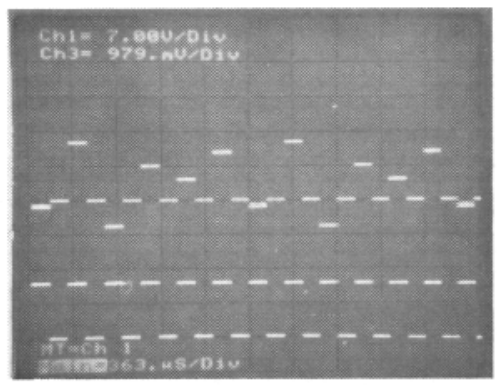

(c)

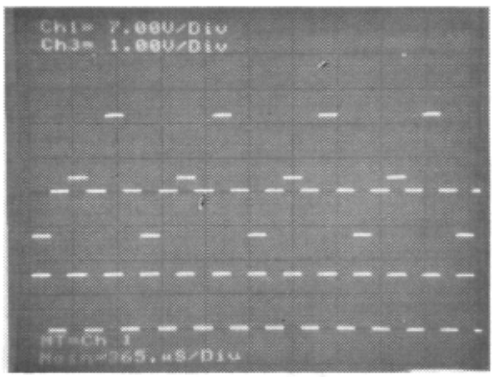

(e)

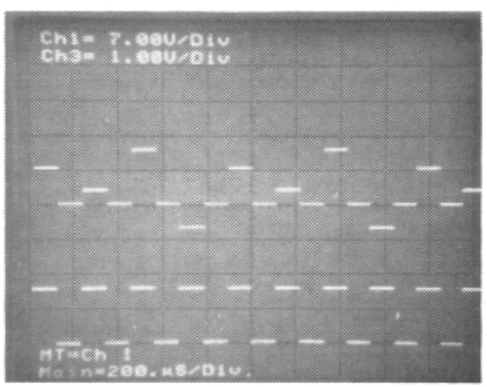

(b)

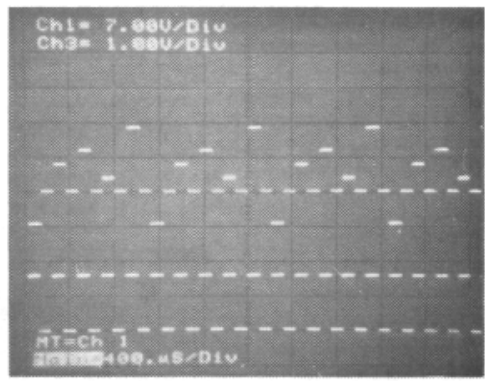

(d)

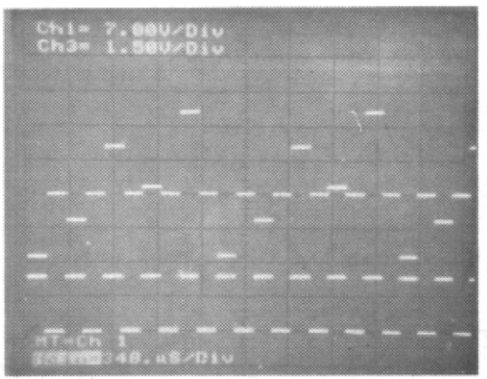

(f)

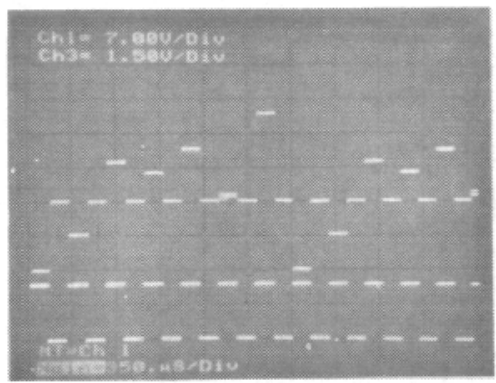

(g)

Fig. 20. Different periodic signals obtained from the circuit of Fig. 18 as the parameter $V$ increases from $0 \mathrm{~V}$. In all of the oscillograms, the lower trace is the clock signal. (a) $V=1.3 \mathrm{~V}$ (period 2). (b) $V=1.6 \mathrm{~V}$ (period 4). (c) $V=1.66 \mathrm{~V}$ (period 6). (d) $V=1.86 \mathrm{~V}$ (period 5). (e) $V=2.24 \mathrm{~V}$ (period 3). (f) $V=3.62 \mathrm{~V}$ (period 5). (g) $V=3.86 \mathrm{~V}$ (period 7). 


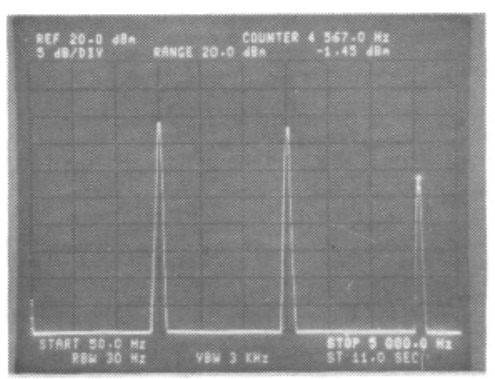

(a)

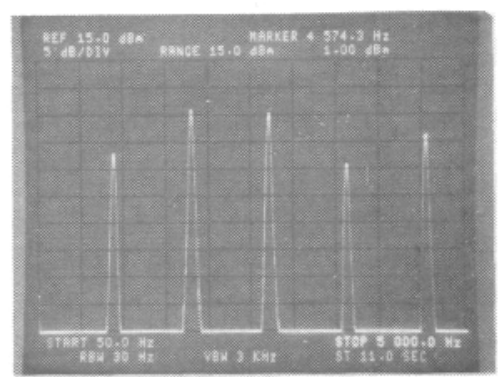

(b)

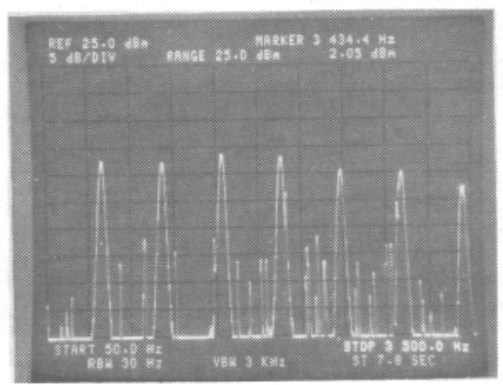

(c)

Fig. 21. Frequency spectra for some odd-period output signals for the circuit of Fig. 18. (a) $V=2.24 \mathrm{~V}$ (period 3). (b) $V=1.86 \mathrm{~V}$ (period 5). (c) $V=3.86 \mathrm{~V}$ (period 7). 


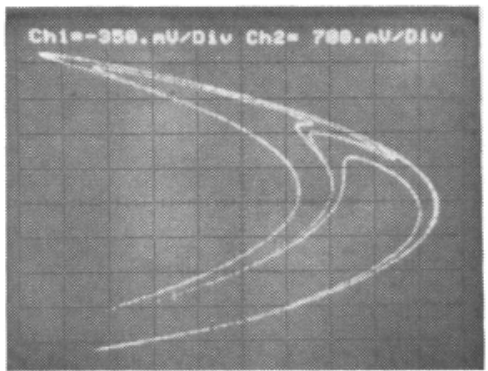

(a)

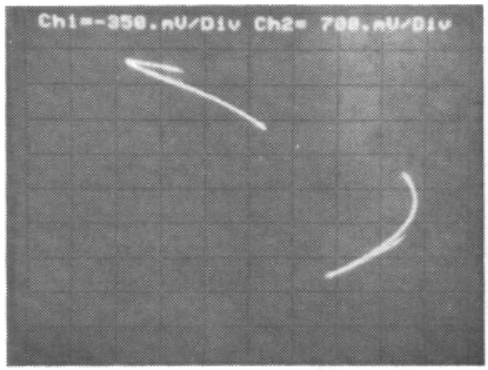

(c)

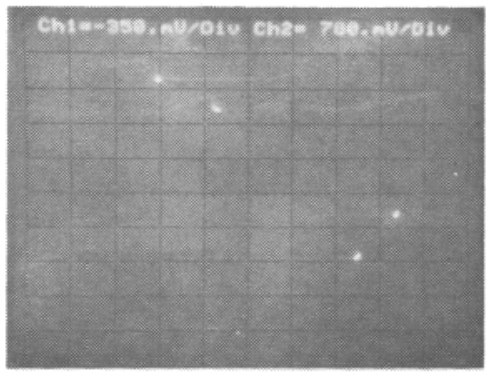

(e)

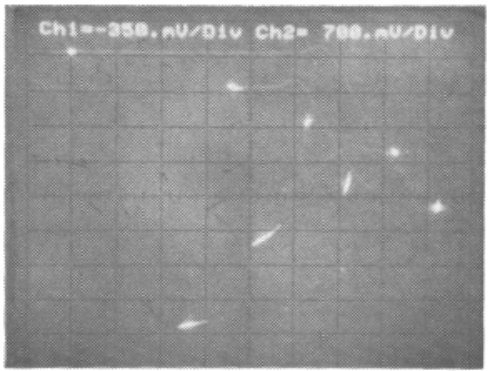

(b)

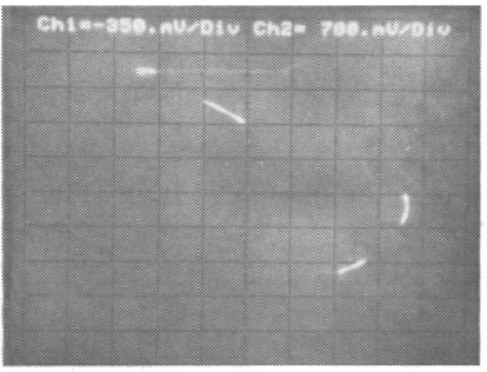

(d)

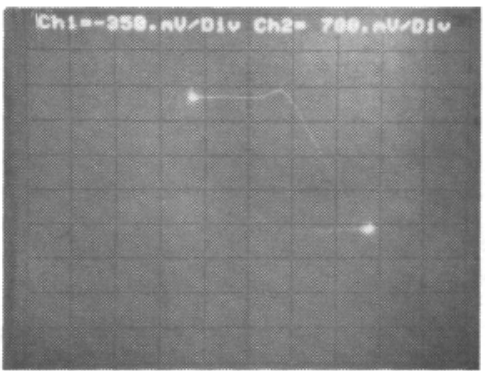

(f)

Fig. 23. Oscilloscope pictures showing several interesting attractors displayed by the circuit in Fig. 12. (a) $V=2.16 \mathrm{~V}$ (the experimental attractor). (b) $V=2.05 \mathrm{~V}$ (8 islets). (c) $V=$ $2 \mathrm{~V}$ (2 islets). (d) $V=1.91 \mathrm{~V}$ (4 islets). (e) $V=1.76 \mathrm{~V}$ (period 4). (f) $V=1.48 \mathrm{~V}$ (period 2).

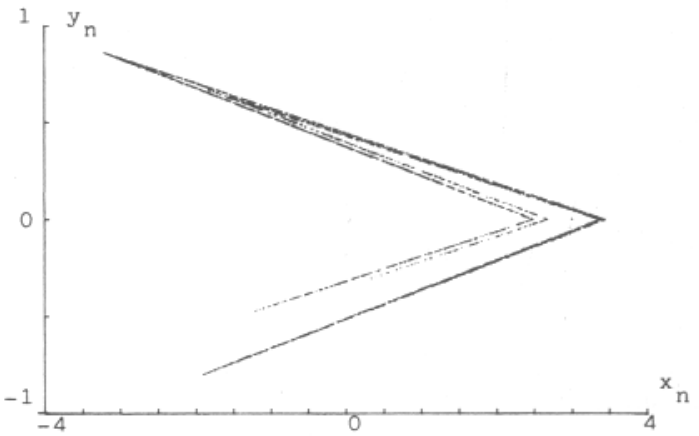

(a)

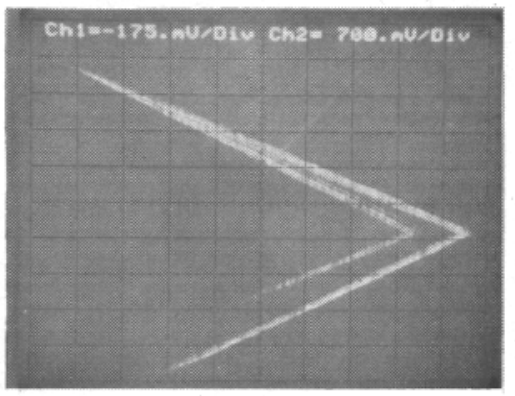

(b)

Fig. 24. (a) Computer simulation of the strange attractor for the map described by (18).

(b) Oscilloscope picture showing the actual attractor displayed by the circuit in Fig. 25. Authorized licensed use limited to: Universidad de Sevilla. Downloaded on April 20,2020 at 15:51:37 UTC from IEEE Xplore. Restrictions apply. 